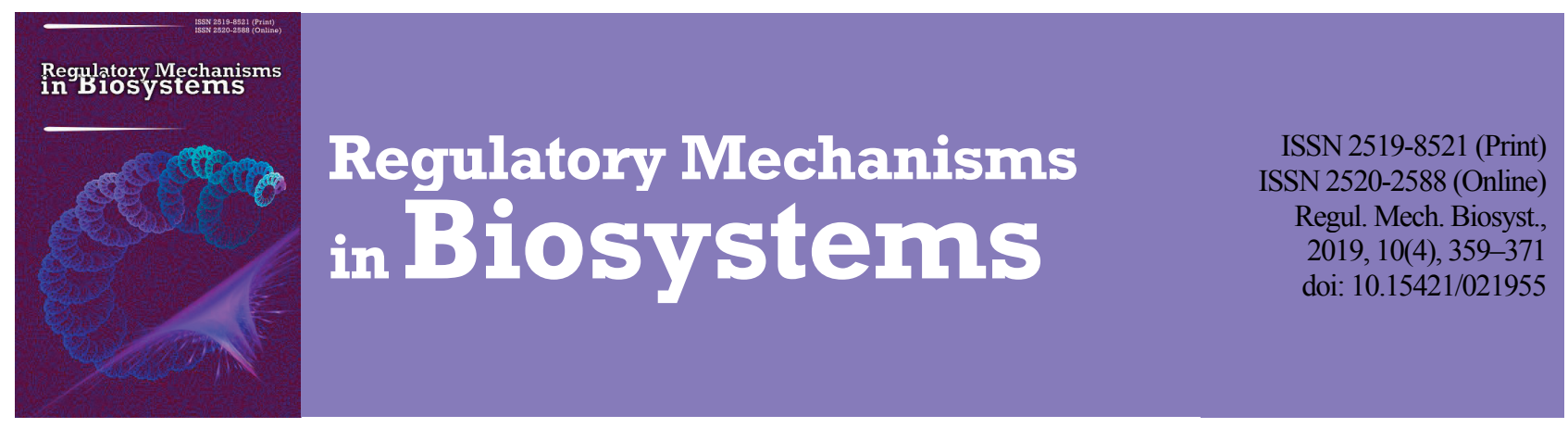

\title{
Impact of essential oil from plants on migratory activity of Sitophilus granarius and Tenebrio molitor
}

\author{
V. O. Martynov, O. Y. Hladkyi, T. M. Kolombar, V. V. Brygadyrenko \\ Oles Honchar Dnipro National University, Dnipro, Ukraine
}

Article info

Received 17.10.2019

Received in revised form 20.11.2019

Accepted 22.11.2019

Oles Honchar Dnipro National University,

Gagarin ave., 72,

Dnipro, 49010, Ukraine.

Tel.: + 38-050-93-90-788.

E-mail:brigad@ua.fm

\begin{abstract}
Martynov, V. O., Hladkyi, O. Y., Kolombar, T. M., \& Brygadyrenko, V. V. (2019). Impact of essential oil from plants on migrato$r y$ activity of Sitophilus granarius and Tenebrio molitor. Regulatory Mechanisms in Biosystems, 10(4), 359-371. doi:10.15421/021955

Measures against pests should be performed in the context of integrated management of agricultural crops and complex control of pests. Therefore, use of ecologically safe approaches is the best option. Essential oils of plants can make an impact on the main metabolic, biochemical, physiological and behavioural functions of insects. We evaluated the effect of 18 essential oils and 18 dried plants on migratory activity of Sitophilus granarius (Linnaeus, 1758) and Tenebrio molitor Linnaeus, 1758 in conditions of laboratory experiment. Notable repellent activity against $S$. granarius was exhibited by Citrus sinensis and Picea abies. Repellent action against T. molitor was displayed by dried and cut leaves of Origanum vulgare and Eucalyptus globulus, and also essential oils from Juniperus communis, P. abies, Pterocarpus santalinus, C. sinensis and C. aurantiifolia. Therefore, out of 18 studied essential oils, only two samples had a notable biological effect on migratory activity of S. granarius and five samples - on T. molitor. These data indicate a possibility of using essential oils or their main components as ecologically safe natural repellents against pests of stored wheat and products of its processing.
\end{abstract}

Keywords: pest control; biopesticides; plant protection; repellent; attractant.

\section{Introduction}

Measures against pests should be performed in the context of integrated management of agricultural crops and complex control of pests, and also should be ecologically-based. Therefore, use of ecologically safe approaches is the most promising variant (Koul \& Walia, 2009). Over the recent 50 years, control of pests in agriculture has been based on use of synthetic chemical insecticides in field agrocenoses and conditions of greenhouse cultivation. However, synthetic insecticides are toxic, they cause a non-favourable impact on the environment, polluting soil, water and air, and also their large scale use leads to development of resistance in the target species and significant damage to populations of non-target species of invertebrates (Benhalima et al., 2004; Pimentel et al., 2009; Boyko \& Brygadyrenko, 2017; Martynov \& Brygadyrenko, 2017, 2018; Martynov et al., 2019). Strict measures of ecological regulation of use of pesticides have led to growth of a number of studies on use of natural plant extracts as an alternative to synthetic preparations (Isman, 2004; Pérez et al., 2010).

There are 17,500 species of aromatic plants and around 300 essential oils, which have commercial importance for cosmetics, pharmaceutics and the food industry (Bakkali et al., 2008; Pushpanathan et al., 2008; Ebadollahi et al., 2015). Over 2,000 species of plants have insecticidal activity (Klocke, 1989). Many commercial essential oils are included in the list Generally Recognized as Safe, fully recognized by the Environmental Protection Agency and Food and Drug Administration of the USA (Burt, 2004).

Essential oils are secondary metabolites and are complex substances which contain many different components that determine the properties of these compounds. Among the constituents of the essential oils, terpenes, aromatic and aliphatic compounds are distinguished. The main terpenes are monoterpenes and sesquiterpenes (Bakkali et al., 2008; Koul et al., 2008). Monoterpenes form up to $90 \%$ of essential oils and are re- presented by compounds different in structure: acyclic (geraniol) and cyclic (terpeniol) spirits, phenols (thymol), ketones (thujone), aldehydes (citronellal), acids (chrysanthemic acid) and oxides (1,8-cineole). Aromatic compounds, such as cinnamaldehyde, chavicol, anethole, safrole and apiole (derivatives from phenylpropane) are present in smaller amount (Isman, 2006; Tripathi et al., 2009).

Essential oils affect the main metabolic, biochemical, physiological and behavioural functions of insects (Mann \& Kaufman, 2012), and can also block airways, leading to asphyxiation and death of pests (Kaufmann \& Briegel, 2004; Rotimi et al., 2011). They can exert toxic, fumigative, repellent, antifeedant, ovicidal, attractant and other actions (Werdin-González et al., 2008). A number of researchers (Isman, 2000; Gutiérrez et al., 2009) report neurotoxic, cytotoxic, phototoxic and mutagenic effects of essential oils on insects. Botanical insecticides have a number of advantages: they do not persist in the environment, they pose relatively low risk for non-target organisms (useful predators and parasites) and are relatively non-toxic for mammals (Weinzierl, 1998; Scott et al., 2003). They usually quickly decompose in the environment and are easely metabolized by the animals that receive sublethal doses (Grdiša \& Gršić, 2013). Reasons for limited commercial use of biological insecticides are their relatively slow action, variable efficiency, absence of stability and non-constant availability compared to synthetic analogues (Isman, 2008). Other obstacles to commercializing botanical insecticides are deficiency of natural resources, difficulties of standardization, control of quality and registration (Isman, 1997).

Sitophilus granarius (Linnaeus, 1758) is one the most harmful and common pests of grain. When feeding, an adult beetle damages different grains and products of its processing. Larvae can develop in grain of wheat, rye, barley, oat, rice, maize, buckwheat, panicgrass, and sometimes live in macaroni products and congealed flour. Another common pest of grain storages is Tenebrio molitor Linnaeus, 1758, which can damage different fractured grains of maize, wheat, soybean and other 
crops (Punzo \& Mutchmor, 1980; Fazolin et al., 2007; Cosimi et al., 2009). Presence of $T$. molitor in stored grain leads to its contamination with enzymes of the body and products of vital activity of the insect, and also contributes to development of saprophytic microflora, reducing the quality of the product (Loudon, 1988; Schroeckenstein et al., 1990; Barnes \& Siva-Jothy, 2000). This insect causes loss of up to $15 \%$ of grain and flour products throughout the world (Dunkel, 1992; Flinn et al., 2003; Neethirajan et al., 2007).

The objective of this article was to evaluate the impact of different essential oils on migratory activity of $S$. granarius and $T$. molitor in the conditions of laboratory experiment.

\section{Materials and methods}

The study on the impact of plant essential oils was conducted in a series of three experiments on two species of insects: S. granarius and T. molitor. In the first experiment, we used imagoes of S. granarius. Before the beginning of the experiment, the animals were kept in a common container with grain of wheat. Insects for the experiment were selected randomly. The experiment was undertaken in polyethylene tubes of $105 \mathrm{~cm}$ length and $2.5 \mathrm{~cm}$ diameter with marks each $10 \mathrm{~cm}$. The tubes were filled with grain of wheat. At one end of the tube, 40 individuals of $S$. granarius and a cotton disk of $0.4 \mathrm{~cm}$ in diameter, saturated with $0.06 \mathrm{~mL}$ of essential oil $-0.48 \mathrm{~mL} / \mathrm{cm}^{2}$ concentration were placed (Table 1). The tubes were closed with a plug at both ends and put randomly on the tables of the laboratory with same illuminance and temperature, out of reach of direct sunlight. After two days, each $10 \mathrm{~cm}$ of the tube with grain were sieved through a laboratory sieve with diameter of cell of $2 \mathrm{~mm}$ for count of weevils in each part of the tube. Each variant of the experiment was performed in five replications. In all variants of the experiment, 1,800 individuals of $S$. granarius were used. The results were statistically analyzed in Statistica 8.0 (Statsoft Inc., USA) software pack using $\chi^{2}$ criterion.

Table 1

Essential oils used in the experiment

on determining migratory activity of Sitophilus granarius

\begin{tabular}{|c|c|c|c|c|c|}
\hline \multirow{2}{*}{$\begin{array}{l}\text { Sub- } \\
\text { stance }\end{array}$} & \multirow[b]{2}{*}{ Plant } & \multicolumn{2}{|c|}{ Chemical composition } & \multirow[b]{2}{*}{ ISO } & \multirow{2}{*}{$\begin{array}{l}\text { Refe- } \\
\text { rences }\end{array}$} \\
\hline & & compounds & $\begin{array}{l}\text { concen- } \\
\text { tration, } \%\end{array}$ & & \\
\hline \multirow{12}{*}{$\begin{array}{l}\text { Tea } \\
\text { tree } \\
\text { oil }\end{array}$} & Melalenca & $\alpha$-pinene & 2.1 & \multirow[t]{12}{*}{4730} & \multirow{12}{*}{$\begin{array}{l}\text { Cox et } \\
\text { al., } 2001\end{array}$} \\
\hline & alternifolia & $\alpha$-terpinene & 8.3 & & \\
\hline & (Maiden \& & $p$-cymene & 2.3 & & \\
\hline & Betche) & limonene & 1.1 & & \\
\hline & Cheel, & 1,8-cineole & 4.5 & & \\
\hline & \multirow[t]{7}{*}{1925} & $\gamma$-terpinene & 17.8 & & \\
\hline & & $\alpha$-terpinolene & 3.3 & & \\
\hline & & terpinen-4-ol & 39.8 & & \\
\hline & & $\alpha$-terpineol & 3.4 & & \\
\hline & & aromadendrene & 1.2 & & \\
\hline & & viridiforene & 1.2 & & \\
\hline & & $\delta$-cadinene & 1.5 & & \\
\hline \multirow{14}{*}{$\begin{array}{l}\text { Euca- } \\
\text { lyptus } \\
\text { oil }\end{array}$} & \multirow{14}{*}{$\begin{array}{l}\text { Eucalyptus } \\
\text { globulus } \\
\text { Labillar- } \\
\text { dière, } 1861\end{array}$} & a-pinene & 5.65 & \multirow[t]{14}{*}{770} & \multirow{14}{*}{$\begin{array}{c}\text { Abdossi } \\
\text { et al., } \\
2015\end{array}$} \\
\hline & & $\beta$-pinene & 0.31 & & \\
\hline & & sabinene & 0.65 & & \\
\hline & & limonene & 0.84 & & \\
\hline & & 1,8-cineole & 76.65 & & \\
\hline & & $\gamma$-terpinene & 0.63 & & \\
\hline & & terpien-4-ol & 0.37 & & \\
\hline & & $\alpha$-terpineol & 1.96 & & \\
\hline & & $\alpha$-terpineol acetate & 4.85 & & \\
\hline & & isoledene & 0.54 & & \\
\hline & & $\alpha$-gurjunene & 0.85 & & \\
\hline & & $\beta$-gurjunene & 0.36 & & \\
\hline & & alloaromadendrene & 3.98 & & \\
\hline & & aromadendrene & 0.51 & & \\
\hline \multirow{7}{*}{$\begin{array}{l}\text { Laven- } \\
\text { der oil }\end{array}$} & \multirow{7}{*}{$\begin{array}{l}\text { Lavandula } \\
\text { angusti- } \\
\text { folia } \\
\text { Miller, } \\
1768\end{array}$} & camphene & 1.37 & \multirow[t]{7}{*}{3515} & \multirow{7}{*}{$\begin{array}{l}\text { Jianu } \\
\text { etal., } \\
2013\end{array}$} \\
\hline & & $\beta$-myrcene & 2.03 & & \\
\hline & & D-limonene & 2.10 & & \\
\hline & & $\beta$-phellandrene & 16.00 & & \\
\hline & & 1,8 -cineole & 15.69 & & \\
\hline & & terpinen-4ol & 9.57 & & \\
\hline & & bomeol & 5.07 & & \\
\hline
\end{tabular}

\begin{tabular}{|c|c|c|c|c|c|}
\hline \multirow{2}{*}{$\begin{array}{l}\text { Sub- } \\
\text { stance }\end{array}$} & \multirow[b]{2}{*}{ Plant } & \multicolumn{2}{|c|}{ Chemical composition } & \multirow[b]{2}{*}{ ISO } & \multirow{2}{*}{$\begin{array}{l}\text { Refe- } \\
\text { rences }\end{array}$} \\
\hline & & compounds & $\begin{array}{l}\text { concen- } \\
\text { tration, \% }\end{array}$ & & \\
\hline & & $\alpha$-terpineol & 6.00 & & \\
\hline & & santalene & 4.50 & & \\
\hline & & caryophyllene & 24.12 & & \\
\hline \multirow{21}{*}{$\begin{array}{l}\text { Melissa } \\
\text { oil }\end{array}$} & \multirow{21}{*}{$\begin{array}{l}\text { Melissa } \\
\text { officinalis } \\
\text { Linnaeus, } \\
1753\end{array}$} & $\alpha$-pinene & 0.05 & \multirow[t]{21}{*}{-} & \\
\hline & & $\beta$-pinene & 0.09 & & etal., \\
\hline & & camphene & 0.12 & & \\
\hline & & myrcene & 0.15 & & \\
\hline & & limonene & 0.74 & & \\
\hline & & $\gamma$-terpinene & 0.40 & & \\
\hline & & p-cymene & 0.83 & & \\
\hline & & octanal & 0.12 & & \\
\hline & & 6-methyl-5-hepten-2-ol & 3.79 & & \\
\hline & & linalool & 0.25 & & \\
\hline & & terpinen-1-o1 & 0.25 & & \\
\hline & & citronellal & 13.32 & & \\
\hline & & $\beta$-caryophyllene & 4.95 & & \\
\hline & & neral & 19.75 & & \\
\hline & & $\alpha$-terpineol & 1.44 & & \\
\hline & & geranial & 26.80 & & \\
\hline & & geranyl acetate & 1.76 & & \\
\hline & & neryl acetate & 1.45 & & \\
\hline & & geraniol & 4.23 & & \\
\hline & & nerol & 0.64 & & \\
\hline & & caryophyllene oxide & 9.99 & & \\
\hline$\overline{\text { Berga- }}$ & Citrus & $\alpha$-thujene & 0.27 & 9800 & Costa \\
\hline mot oil & bergamia & $\alpha$-pinene & 1.04 & & etal., \\
\hline & Risso \& & sabinene & 0.89 & & 2010 \\
\hline & Poiteau, & $\beta$-pinene & 5.59 & & \\
\hline & (1819) & myrcene & 0.91 & & \\
\hline & & $\alpha$-terpinene & 0.11 & & \\
\hline & & p-cymene & 0.37 & & \\
\hline & & limonene & 42.80 & & \\
\hline & & (Z)- $\beta$-ocimene & 0.17 & & \\
\hline & & $\gamma$-terpinene & 6.19 & & \\
\hline & & terpinolene & 0.24 & & \\
\hline & & linalool & 5.55 & & \\
\hline & & octyl acetate & 0.10 & & \\
\hline & & neral & 0.16 & & \\
\hline & & linalyl acetate & 27.14 & & \\
\hline & & geranial & 0.24 & & \\
\hline & & $\alpha$-terpinyl acetate & 0.14 & & \\
\hline & & neryl acetate & 0.30 & & \\
\hline & & geranyl acetate & 0.31 & & \\
\hline & & (E)-caryophyllene & 0.25 & & \\
\hline & & trans- $\alpha$-bergamotene & 0.25 & & \\
\hline & & $\beta$-bisabolene & 0.36 & & \\
\hline Orange & Citrus & $\alpha$-pinene & 0.36 & 3140 & Singh \\
\hline oil & sinensis & sabinine & 0.37 & & etal., \\
\hline & (Linnaeus) & $\beta$-myrcene & 1.71 & & 2010 \\
\hline & Osbeck & octanal & 0.43 & & \\
\hline & (pro.sp.) & limonene & 90.66 & & \\
\hline & & linalylacetate & 2.80 & & \\
\hline & & t-sabinine hydrate & 0.42 & & \\
\hline & & laevo- $\beta$-pinene & 0.46 & & \\
\hline & & geranyl formate & 0.65 & & \\
\hline Grape- & Citrus & $\alpha$-pinene & 0.4 & 3053 & Uysal \\
\hline fruitoil & paradisi & sabinene & 0.3 & & etal., \\
\hline & Macfa- & $\beta$-pinene & 0.8 & & 2011 \\
\hline & dyen, 1830 & $\beta$-myrcene & 0.7 & & \\
\hline & & $\alpha$-terpinene & 0.7 & & \\
\hline & & limonene & 91.5 & & \\
\hline & & linalool & 1.1 & & \\
\hline & & trans-limonene oxide & 0.9 & & \\
\hline & & citronellal & 0.4 & & \\
\hline & & $\alpha$-terpineol & 0.3 & & \\
\hline & & nerol & 0.3 & & \\
\hline & & neral & 0.4 & & \\
\hline & & geraniol & 0.3 & & \\
\hline & & geranial & 0.4 & & \\
\hline Spruce & Picea & santene & 2.27 & - & Radu- \\
\hline & abies & $\alpha$-thujene & 0.74 & & lescu \\
\hline & (Linnaeus) & $\alpha$-pinene & 5.40 & & etal., \\
\hline & H. Kar- & camphene & 7.55 & & 2011 \\
\hline & sten., 1881 & $\beta$-pinene & 0.52 & & \\
\hline
\end{tabular}




\begin{tabular}{|c|c|c|c|c|c|}
\hline \multirow{2}{*}{$\begin{array}{l}\text { Sub- } \\
\text { stance }\end{array}$} & \multirow[b]{2}{*}{ Plant } & \multicolumn{2}{|c|}{ Chemical composition } & \multirow[b]{2}{*}{ ISO } & \multirow[b]{2}{*}{$\begin{array}{l}\text { Refe- } \\
\text { rences }\end{array}$} \\
\hline & & compounds & $\begin{array}{c}\text { concen- } \\
\text { tration, } \%\end{array}$ & & \\
\hline & & $\beta$-myrcene & 0.75 & & \\
\hline & & limonene & 9.29 & & \\
\hline & & 1,8-cineole & 0.45 & & \\
\hline & & camphor & 0.40 & & \\
\hline & & borneol & 1.11 & & \\
\hline & & bornyl acetate & 11.78 & & \\
\hline & & $\alpha$-humulene & 0.60 & & \\
\hline & & $\gamma$-muurolene & 0.46 & & \\
\hline & & $\beta$-selinene & 0.42 & & \\
\hline & & $\alpha$-muurolene & 1.61 & & \\
\hline & & $\gamma$-cadinene & 1.54 & & \\
\hline & & $\delta$-cadinene & 9.49 & & \\
\hline & & nerolidol & 1.01 & & \\
\hline & & 1-epi-cubenol & 0.52 & & \\
\hline & & $\alpha$-muurolol & 11.01 & & \\
\hline & & $\delta$-cadinol & 1.48 & & \\
\hline & & $\alpha$-cadinol & 21.39 & & \\
\hline & & manool & 3.58 & & \\
\hline
\end{tabular}

Note: $*$-number of ISO standard.

In the second and the third experiments, we used third age larvae of T. molitor. For one month before the beginning of the experiments, they were kept in a general container and fed with a single component diet (dry rolled oats). Larvae for experiments were selected randomly. The second experiment was undertaken in polyethylene tubes of $4 \mathrm{~cm}$ diameter and $105 \mathrm{~cm}$ length with marks each $10 \mathrm{~cm}$. In one part of the tube, $400 \mathrm{~g}$ of wheat grain with cut up plants was placed (Table 2), and $400 \mathrm{~g}$ of grain with no additions was put in the other part. At $10 \mathrm{~cm}$ intervals along the tube, 5 larvae of $T$. molitor were placed. The tubes were put randomly on the tables in the laboratory with same illuminance and temperature, out of reach of direct sunlight. After two days, from each $10 \mathrm{~cm}$ of the tube, grain was extracted and sieved through a laboratory sieve with cells of $2 \mathrm{~mm}$ in diameter for detecting and counting larvae of T. molitor in each section of the tube. Each variant of the experiment was performed in ten replications. In all variants of the experiment, 9,500 individuals of $T$. molitor (9,000 in 18 variants of the experiment and 500 in the control) were used.

Table 2

Plants used in the experiment on determining migratory activity of $T$. molitor

\begin{tabular}{|c|c|c|c|c|c|}
\hline \multirow[b]{2}{*}{$\begin{array}{l}\text { Common } \\
\text { name }\end{array}$} & \multirow{2}{*}{$\begin{array}{l}\text { Latin } \\
\text { name }\end{array}$} & \multirow{2}{*}{$\begin{array}{c}\text { Content of } \\
\text { essential } \\
\text { oils, } \%\end{array}$} & \multicolumn{2}{|c|}{ Chemical composition } & \multirow[b]{2}{*}{ References } \\
\hline & & & substances & $\begin{array}{c}\text { concen- } \\
\text { tration, } \%\end{array}$ & \\
\hline $\begin{array}{l}\text { Tasma- } \\
\text { nian } \\
\text { bluegum }\end{array}$ & $\begin{array}{l}\text { Euca- } \\
\text { lyptus } \\
\text { globulus } \\
\text { Labillar- } \\
\text { dière, } \\
1861\end{array}$ & $1.6-3.0$ & $\begin{array}{l}\text { a-pinene } \\
\beta \text {-pinene } \\
\text { sabinene } \\
\text { limonene } \\
1,8 \text {-cineole } \\
\text { cis- } \beta \text {-ocimene } \\
\gamma \text {-terpinene } \\
\text { terpien-4-ol } \\
\alpha \text {-terpineol } \\
\alpha \text {-terpineol acetate } \\
\text { isoledene } \\
\alpha \text {-gurjunene } \\
\beta \text {-gurjunene } \\
\text { alloaromadendrene } \\
\text { aromadendrene }\end{array}$ & $\begin{array}{r}5.65 \\
0.31 \\
0.65 \\
0.84 \\
76.65 \\
0.15 \\
0.63 \\
0.37 \\
1.96 \\
4.85 \\
0.54 \\
0.85 \\
0.36 \\
3.98 \\
0.51 \\
\end{array}$ & $\begin{array}{c}\text { Chalchat } \\
\text { etal., 1995; } \\
\text { Abdossi } \\
\text { et al., } 2015\end{array}$ \\
\hline Lavender & $\begin{array}{l}\text { Lavan- } \\
\text { dula } \\
\text { angusti- } \\
\text { folia } \\
\text { Miller, } \\
1768\end{array}$ & $1.13-2.75$ & $\begin{array}{l}\alpha \text {-thujene } \\
\alpha \text {-pinene } \\
\text { camphene } \\
\text { sabinene } \\
\beta \text {-pinene } \\
\beta \text {-myrcene } \\
\text { carene } \\
\text { D-limonene } \\
\beta \text {-phellandrene } \\
1,8 \text {-cineole } \\
\gamma \text {-terpinene } \\
\text { terpinen-4-ol } \\
\text { bomeol }\end{array}$ & $\begin{array}{r}0.40 \\
0.78 \\
1.37 \\
0.31 \\
0.94 \\
2.03 \\
0.76 \\
2.10 \\
16.00 \\
15.69 \\
0.48 \\
9.57 \\
5.07 \\
\end{array}$ & $\begin{array}{c}\text { Jianu et al., } \\
2013\end{array}$ \\
\hline
\end{tabular}

\begin{tabular}{|c|c|c|c|c|c|}
\hline \multirow[b]{2}{*}{$\begin{array}{c}\text { Common } \\
\text { name }\end{array}$} & \multirow[b]{2}{*}{$\begin{array}{l}\text { Latin } \\
\text { name }\end{array}$} & \multirow{2}{*}{$\begin{array}{c}\text { Content of } \\
\text { essential } \\
\text { oils, } \%\end{array}$} & \multicolumn{2}{|c|}{ Chemical composition } & \multirow[b]{2}{*}{ References } \\
\hline & & & substances & $\begin{array}{l}\text { concen- } \\
\text { tration, } \%\end{array}$ & \\
\hline & & & $\alpha$-terpineol & 6.00 & \\
\hline & & & santalene & 4.50 & \\
\hline & & & caryophyllene & 24.12 & \\
\hline & & & $\beta$-sesquiphellandrene & 0.39 & \\
\hline \multirow[t]{21}{*}{$\begin{array}{l}\text { Lemon } \\
\text { balm }\end{array}$} & \multirow{21}{*}{$\begin{array}{l}\text { Melissa } \\
\text { officinalis } \\
\text { Linnaeus, } \\
1753\end{array}$} & \multirow[t]{21}{*}{2.36} & $\begin{array}{l}\text { propanoic acid } \\
\text { propanoic acid 2- }\end{array}$ & 1.93 & \multirow[t]{21}{*}{$\begin{array}{l}\text { Pereira et al., } \\
2014\end{array}$} \\
\hline & & & hydroxy butyl ester & 1.98 & \\
\hline & & & 2,5-pyrrolidinedione & 1.04 & \\
\hline & & & ethyl hydrogen succinate & 3.88 & \\
\hline & & & conhydrin & 1.62 & \\
\hline & & & ethyl iso-allocholate & 5.06 & \\
\hline & & & heptatriacotonol & 1.33 & \\
\hline & & & gallic acid & 9.54 & \\
\hline & & & hexadecanoic acid & 1.97 & \\
\hline & & & hexadecanoic acid & 109 & \\
\hline & & & $\beta$-sitosterol & 45.59 & \\
\hline & & & octadecatrienoic acid & & \\
\hline & & & methyl ester & 2.11 & \\
\hline & & & chlorogenic acid & 1.81 & \\
\hline & & & linoleic acid ethyl ester & 0.99 & \\
\hline & & & hexadecanoic acid butyl & & \\
\hline & & & ester & 2.97 & \\
\hline & & & lupeol & 0.98 & \\
\hline & & & caffeic acid & 3.07 & \\
\hline & & & caffeine & 1.26 & \\
\hline & & & octanal & 2.10 & \\
\hline Pepper- & Mentha & 2.50 & $\alpha$-pinene & 0.32 & Andoğan \\
\hline $\operatorname{mint}$ & piperita & & sabinene & 0.26 & et al., 2002; \\
\hline & Linnaeus, & & $\beta$-pinene & 0.58 & Saharkhiz \\
\hline & 1753 & & 1,8-cineole & 6.69 & etal., 2012 \\
\hline & & & cis-sabinene hydrate & 0.50 & \\
\hline & & & menthone & 2.45 & \\
\hline & & & menthofuran & 11.18 & \\
\hline & & & neomenthol & 2.79 & \\
\hline & & & menthol & 53.28 & \\
\hline & & & neomenthyl acetate & 0.65 & \\
\hline & & & menthyl acetate & 15.10 & \\
\hline & & & isomenthyl acetate & 0.61 & \\
\hline & & & $\beta$-bourbonene & 0.37 & \\
\hline & & & (Z)-caryophyllene & 2.06 & \\
\hline & & & $E$ - $\beta$-farmesene & 0.30 & \\
\hline & & & germacrene D & 2.01 & \\
\hline & & & bicyclogermacrene & 0.22 & \\
\hline Absin- & Artemisia & 1.30 & sabinene & 1.6 & Rezaeinodehi \\
\hline thium & absin- & & myrcene & 10.8 & \& Khangholi, \\
\hline & thium & & $\alpha$-phellandrene & 0.8 & 2008 \\
\hline & Linnaeus, & & para-cymene & 1.2 & Lopes-Lutz \\
\hline & 1753 & & 1,8-cineole & 1.0 & et al., 2008 \\
\hline & & & (Z)- $\beta$-ocimene & 1.5 & \\
\hline & & & (E)- $\beta$-ocimene & 0.5 & \\
\hline & & & linalool & 4.6 & \\
\hline & & & cis-thujone & 0.5 & \\
\hline & & & trans-thujone & 10.1 & \\
\hline & & & (Z)-myroxide & 2.4 & \\
\hline & & & terpinen-4-ol & 1.7 & \\
\hline & & & trans-sabinyl acetate & 26.4 & \\
\hline & & & $\beta$-caryophyllene & 0.9 & \\
\hline & & & neryl isovalerate & 1.8 & \\
\hline Bay tree & Laurus & 1.86 & $\alpha$-pinene & 6.1 & Dadalioğlu \& \\
\hline & nobilis & & sabinene & 12.1 & Evrendilek, \\
\hline & Linnaeus, & & $\beta$-terpinene & 0.1 & 2004; Derwich \\
\hline & 1753 & & 1,8-cineole & 60.7 & et al., 2009 \\
\hline & & & $\gamma$-terpinene & 1.0 & \\
\hline & & & linalool & 0.7 & \\
\hline & & & terpinen-4-ol & 3.3 & \\
\hline & & & $\alpha$-terpineol & 2.0 & \\
\hline & & & $\alpha$-terpinene & 12.5 & \\
\hline & & & eugenol & 0.5 & \\
\hline & & & $\beta$-caryophyllene & 0.4 & \\
\hline & & & methyl eugenol & 0.7 & \\
\hline Breck- & Thymus & 1.05 & $\alpha$-thujene & 1.1 & Lee et al., 2005; \\
\hline land & serpyllum & & $\alpha$-pinene & 2.0 & Nikolić et al., \\
\hline thyme & Linnaeus, & & camphene & 2.4 & 2014 \\
\hline & 1753 & & sabinene & 0.8 & \\
\hline
\end{tabular}




\begin{tabular}{|c|c|c|c|c|c|c|c|c|c|c|c|}
\hline \multirow{2}{*}{$\begin{array}{c}\text { Common } \\
\text { name }\end{array}$} & \multirow[b]{2}{*}{$\begin{array}{l}\text { Latin } \\
\text { name }\end{array}$} & \multirow{2}{*}{$\begin{array}{c}\text { Content of } \\
\text { essential } \\
\text { oils, } \%\end{array}$} & \multicolumn{2}{|c|}{ Chemical composition } & \multirow[b]{2}{*}{ References } & & & Content of & Chemical com & ition & \\
\hline & & & substances & $\begin{array}{c}\text { concen- } \\
\text { tration, } \%\end{array}$ & & name & name & $\begin{array}{c}\text { essential } \\
\text { oils, } \%\end{array}$ & substances & $\begin{array}{c}\text { concen- } \\
\text { tration, } \%\end{array}$ & References \\
\hline & & & $\beta$-myrcene & 1.3 & & & & & bornyl acetate & 1.9 & \\
\hline & & & $\alpha$-terpinene & 1.1 & & Tansy & Tanace- & 0.22 & tricyclene & 0.1 & Schearer, \\
\hline & & & p-cymene & 8.9 & & & tum & & $\alpha$-pinene & 0.4 & 1984 \\
\hline & & & limonene & 0.6 & & & vulgare & & camphene & 2.5 & \\
\hline & & & $\gamma$-terpinene & 7.2 & & & Linnaeus, & & sabinene & 6.0 & \\
\hline & & & cis-sabinene hydrate & 0.5 & & & 1753 & & $\alpha$-terpinene & 0.1 & \\
\hline & & & linalool & 2.4 & & & & & 1,8 -cineole & 5.1 & \\
\hline & & & camphor & 0.7 & & & & & $\gamma$-terpinene & 0.5 & \\
\hline & & & borneol & 6.0 & & & & & $\rho$-cymene & 1.0 & \\
\hline & & & terpinene-4-ol & 0.7 & & & & & sabinol acetate & 0.2 & \\
\hline & & & thymol methyl ether & 3.8 & & & & & camphor & 29.6 & \\
\hline & & & bornyl acetate & 7.0 & & & & & terpinen-4-ol & 1.5 & \\
\hline & & & thymol & 38.5 & & & & & umbellulone & 24.7 & \\
\hline & & & carvacrol & 4.7 & & & & & bomeol & 0.8 & \\
\hline & & & thymol acetate & 2.8 & & & & & carvone & 8.3 & \\
\hline & & & $\beta$-caryophyllene & 1.3 & & & & & valeranone & 6.8 & \\
\hline & & & $\beta$-bisabolene & 1.0 & & & & & thymol & 10.3 & \\
\hline & & & eudesm-3-en-6-ol & 0.6 & & Immor- & Helich- & 0.09 & linalool & 1.7 & Czinner \\
\hline Rosema- & Rosma- & 1.90 & $\alpha$-pinene & 14.90 & Özcan \& & telle & rysum & & $\alpha$-terpineol & 1.8 & et al., 2000 \\
\hline ry & rinus & & camphene & 3.33 & Chalchat, 2008; & & arenarium & & octanoic acid & 6.0 & \\
\hline & officinalis & & $\beta$-octanone & 1.61 & Gachkar et al., & & Moench, & & carvone & 1.1 & \\
\hline & Linnaeus, & & sabinene & 0.56 & 2007 & & 1794 & & anethole & 3.2 & \\
\hline & 1753 & & myrcene & 2.07 & & & & & nonanoic acid & 6.9 & \\
\hline & & & O-cymene & 0.71 & & & & & $\beta$-caryophyllene & 0.6 & \\
\hline & & & 1,8-cineole & 7.43 & & & & & thymol & 0.6 & \\
\hline & & & linalool & 14.90 & & & & & carvacrol & 3.6 & \\
\hline & & & myrcenol & 0.75 & & & & & $\alpha$-humulene & 0.5 & \\
\hline & & & camphor & 4.97 & & & & & eugenol & 0.4 & \\
\hline & & & borneol & 3.68 & & & & & decanoic acid & 9.8 & \\
\hline & & & terpinen-4-ol & 1.70 & & & & & $\delta$-cadinene & 0.7 & \\
\hline & & & $\alpha$-terpineol & 0.83 & & & & & butylhydroxyanisole & 0.6 & \\
\hline & & & verbinone & 1.94 & & & & & dodecanoic acid & 11.9 & \\
\hline & & & piperitone & 23.70 & & & & & $\alpha$-muurolol & 1.3 & \\
\hline & & & bomyl acetate & 3.08 & & & & & $\beta$-asarone & 1.5 & \\
\hline & & & $\beta$-caryophyllene & 2.68 & & & & & globulol & 1.4 & \\
\hline & & & cis- $\beta$-farnesene & 1.26 & & & & & methyl palmitate & 28.5 & \\
\hline & & & germacrene D & 0.52 & & $\overline{\text { Sage }}$ & Salvia & 1.11 & $\alpha$-pinene & 2.6 & Perry et al., \\
\hline & & & $\alpha$-bisabolol & 1.01 & & & officinalis & & camphene & 2.1 & 1999 \\
\hline Cloves & Syzygium & 3.00 & p-cymene & 0.90 & Nassar & & Linnaeus, & & $\beta$-pinene & 6.0 & \\
\hline & aroma- & & 5-hexene-2-one & 0.67 & et al., 2007; & & 1753 & & myrcene & 0.9 & \\
\hline & ticum & & thymol & 0.87 & Lee etal., 2009 & & & & 1,8-cineole & 9.2 & \\
\hline & (Linna- & & eugenol & 71.56 & & & & & (Z)-ocimene & 0.4 & \\
\hline & eus) & & eugenyl acetate & 8.99 & & & & & $\alpha$-thujone & 34.6 & \\
\hline & Merrill & & caryophyllene oxide & 1.67 & & & & & $\beta$-thujone & 5.0 & \\
\hline & etPerry, & & guaiol & 0.90 & & & & & camphor & 6.5 & \\
\hline & 1989 & & nootkatin & 1.05 & & & & & borneol & 2.4 & \\
\hline & & & isolongifolanone & 0.86 & & & & & bornyl acetate & 0.4 & \\
\hline & & & hexadecanoic acid & 0.50 & & & & & $\beta$-caryophyllene & 4.5 & \\
\hline & & & 9,17-octadecadienal & 0.24 & & & & & aromadendrene & 0.5 & \\
\hline & & & octadecanoicacid butylester & 0.33 & & & & & $\alpha$-humulene & 6.2 & \\
\hline & & & vitamin E acetate & 0.43 & & & & & germacrene D & 0.3 & \\
\hline Yarrow & Achillea & $0.13-0.34$ & thymol & 0.5 & Pino & & & & $\delta$-cadinene & 0.4 & \\
\hline & millefo- & & ethyl nonanoate & 2.6 & etal., 1998; & & & & caryophyllene oxide & 0.7 & \\
\hline & lium & & sabinene & 5.4 & Rohloff & & & & viridiflorol & 4.5 & \\
\hline & Linnaeus, & & $\beta$-caryophyllene & 5.2 & et al., 2000 & & & & $\alpha$-humulene oxide & 0.9 & \\
\hline & 1753 & & ethyl hexanoate & 0.6 & & & & & manool & 1.1 & \\
\hline & & & $\alpha$-humulene & 0.7 & & Oregano & Origanum & 2.50 & $\alpha$-thujene & 2.2 & Mechergui \\
\hline & & & p-cymene & 0.6 & & & vulgare & & $\alpha$-pinene & 0.7 & et al., 2010; \\
\hline & & & 1,8-cineole & 5.7 & & & Linnaeus, & & sabinene & 1.0 & Teixeira \\
\hline & & & germacrene D & 0.8 & & & 1753 & & $\beta$-myrcene & 1.3 & etal., 2013 \\
\hline & & & viridiflorene & 0.8 & & & & & $\alpha$-terpinene & 3.7 & \\
\hline & & & linalool & 1.0 & & & & & $\beta$-phellandrene & 0.9 & \\
\hline & & & camphor & 1.2 & & & & & cis- $\beta$-ocimene & 1.6 & \\
\hline & & & hurnulene epoxide II & 3.2 & & & & & trans- $\beta$-ocimene & 1.5 & \\
\hline & & & bomeol & 9.8 & & & & & $\gamma$-terpinene & 11.6 & \\
\hline & & & 14hydroxy- $\alpha$-muurolene & 0.8 & & & & & $\alpha$-terpinolene & 0.9 & \\
\hline & & & terpinen-4-ol & 2.8 & & & & & $\beta$-bisabolene & 2.1 & \\
\hline & & & $\alpha$-terpineol & 2.0 & & & & & linalool & 2.6 & \\
\hline & & & methyl hexadecanoate & 0.8 & & & & & menthone & 0.7 & \\
\hline & & & caryophyllene oxide & 20.0 & & & & & $\delta$-terpineol & 7.5 & \\
\hline & & & (E)-isoeugenyl acetate & 1.1 & & & & & $\beta$-fenchyl alcohol & 12.8 & \\
\hline & & & ethyl octanoate & 1.5 & & & & & pulegone & 1.0 & \\
\hline & & & hexadecanoic acid & 1.1 & & & & & carvacrol & 14.5 & \\
\hline & & & pulegone & 2.4 & & & & & spathulenol & 0.5 & \\
\hline
\end{tabular}




\begin{tabular}{|c|c|c|c|c|c|c|c|c|c|c|c|}
\hline \multirow{2}{*}{$\begin{array}{c}\text { Common } \\
\text { name }\end{array}$} & \multirow{2}{*}{$\begin{array}{l}\text { Latin } \\
\text { name }\end{array}$} & \multirow{2}{*}{$\begin{array}{c}\text { Content of } \\
\text { essential } \\
\text { oils, } \%\end{array}$} & \multicolumn{2}{|c|}{ Chemical composition } & \multirow[b]{2}{*}{ References } & \multirow{2}{*}{$\begin{array}{c}\text { Common } \\
\text { name }\end{array}$} & \multirow{2}{*}{$\begin{array}{l}\text { Latin } \\
\text { name }\end{array}$} & \multirow{2}{*}{$\begin{array}{c}\text { Content of } \\
\text { essential } \\
\text { oils, } \%\end{array}$} & \multicolumn{2}{|c|}{ Chemical composition } & \multirow[b]{2}{*}{ References } \\
\hline & & & substances & $\begin{array}{l}\text { concen- } \\
\text { tration, } \%\end{array}$ & & & & & substances & $\begin{array}{l}\text { concen- } \\
\text { tration, } \%\end{array}$ & \\
\hline & & & caryophyllene oxide & 0.6 & & & & & $\alpha$-terpineol & 1.0 & \\
\hline & & & thymol & 12.6 & & & & & cis-geraniol & 1.0 & \\
\hline \multirow{25}{*}{$\begin{array}{l}\text { Wild } \\
\text { rosemary }\end{array}$} & Rhodo- & $0.14-0.87$ & $\alpha$-pinene & 0.7 & Raal etal., & & & & $\beta$-caryophyllene & 1.4 & \\
\hline & dendron & & $\beta$-pinene & 0.5 & 2014 & & & & $\alpha$-bergamotene & 7.6 & \\
\hline & tomen- & & $\beta$-myrcene & 1.3 & & & & & $\gamma$-muurolene & 0.7 & \\
\hline & tosum & & p-cymene & 12.5 & & & & & germacrene D & 2.0 & \\
\hline & Harmaja & & $\rho$-cymenene & 0.5 & & & & & bicyclogermacrene & 0.8 & \\
\hline & $(1990)$ & & (E)-pinocarveol & 0.7 & & & & & $\gamma$-cadinene & 4.9 & \\
\hline & & & lepalin & 0.7 & & & & & calamenene & 0.7 & \\
\hline & & & pinocarvone & 0.8 & & & & & spathulenol & 0.5 & \\
\hline & & & $\alpha$-thujenal & 0.4 & & & & & viridiflorol & 1.6 & \\
\hline & & & p-cymen-8-ol & 0.7 & & & & & epi- $\alpha$-cadinol & 10.0 & \\
\hline & & & myrtenal & 0.8 & & Camo- & Matrica- & $0.62-0.75$ & $p$-cymene & 0.11 & Pirzad \\
\hline & & & $\gamma$-terpineol & 31.2 & & mile & ria cha- & & limonene & 0.10 & et al., 2006; \\
\hline & & & piperitone & 0.7 & & & momilla & & trans- $\beta$-ocimene & 0.11 & Heuskin \\
\hline & & & lepalone & 1.3 & & & Linnaeus, & & cis- $\alpha$-ocimene & 0.69 & et al., 2009 \\
\hline & & & isopiperitenone & 0.4 & & & 1753 & & $\gamma$-terpinene & 0.17 & \\
\hline & & & lepalol & 2.6 & & & & & artemesia ketone & 0.32 & \\
\hline & & & isoascaridol & 2.7 & & & & & $\alpha$-isocomene & 0.26 & \\
\hline & & & alloaromadendrene & 0.6 & & & & & $\beta$-caryophyllene & 0.17 & \\
\hline & & & ledene & 0.4 & & & & & $E$ - $\beta$-famesene & 42.59 & \\
\hline & & & palustrol & 15.9 & & & & & germacrène D & 2.93 & \\
\hline & & & epiglobulol & 0.7 & & & & & $\beta$-selinene & 0.22 & \\
\hline & & & ledol & 11.8 & & & & & $(Z, E)$ - $\alpha$-farnesene & 0.83 & \\
\hline & & & $\beta$-oplopenone & 0.4 & & & & & bicyclogermacrene & 1.99 & \\
\hline & & & (Z)-nerolidol acetate & 2.1 & & & & & $(E, E)$ - $\alpha$-famesene & 8.32 & \\
\hline & & & cyclocolorenone & 1.6 & & & & & $\delta$-cadinene & 0.18 & \\
\hline \multirow{13}{*}{$\begin{array}{l}\text { European } \\
\text { birthwort }\end{array}$} & Aristo- & 1.10 & 2-hexanone & 1.6 & Dhouioui & & & & sesquirosefuran & 0.18 & \\
\hline & lochia & & propyl-2-valerate & 6.3 & et al., 2016 & & & & trans-nerolidol & 0.17 & \\
\hline & clematitis & & butyl isobutyrate & 8.0 & & & & & dehydronerolidol & 0.09 & \\
\hline & Linnaeus, & & 1,8-cineole & 1.2 & & & & & dendrolasin & 0.21 & \\
\hline & 1753 & & artemisia ketone & 2.0 & & & & & spathulenol & 0.63 & \\
\hline & & & phenyl ethyl alcohol & 1.5 & & & & & globulol & 0.23 & \\
\hline & & & linalool & 1.0 & & & & & $\alpha$-bisabolol oxide B & 4.43 & \\
\hline & & & camphor & 2.2 & & & & & $\alpha$-bisabolone oxide $\mathrm{A}$ & 4.53 & \\
\hline & & & trans-pinocarveol & 1.4 & & & & & chamazulene & 1.18 & \\
\hline & & & cis-verbenol & 1.1 & & & & & $\alpha$-bisabolol oxide A & 21.16 & \\
\hline & & & trans-verbenol & 2.2 & & & & & cis-eneyne-dicyclo ether & 5.94 & \\
\hline & & & terpinen-4-ol & 2.7 & & & & & trans-eneyne-dicyclo ether & 0.99 & \\
\hline & & & terpineol & 1.5 & & & & & (E)-phytol & 0.23 & \\
\hline
\end{tabular}

verbenone 2.1

bornyl acetate $\quad 1.2$

methyl myitenate $\quad 2.5$

$\beta$-elemene $\quad 4.7$

trans- $\beta$-caryophyllene $\quad 4.4$

valerena 4,7(11)-diene $\quad 3.5$

allo-aromadendrene $\quad 1.3$

bicyclosesquiphellandrene $\quad 1.5$

valencene 1.5

paciforgiol $\quad 3.2$

$\alpha$-elemol $\quad 3.1$

epi-globulol $\quad 10.0$

calarene oxide $\quad 4.9$

maaliol 1.2

caryophyllene-oxide $\quad 4.6$

$\beta$-funebrene epoxide $\quad 4.4$

aromadendrene oxide $\quad 14.0$

$\beta$-atlantol $\quad 5.7$

aromadendreneoxide $\quad 3.2$

isospathulenol $\quad 1.8$

T-cadinol $\quad 2.1$

agarospirol $\quad 1.6$

$\tau$-muurolol 1.5

bisabolone-oxide A $\quad 2.9$

$\beta$-elemene dioxide $\quad 2.2$

eremophilone cyclocolo- $\quad 8.4$

renone $\quad 3.6$

14-oxy- $\alpha$-muurolene $\quad 1.3$

$\alpha$-vetivone $\quad 1.4$

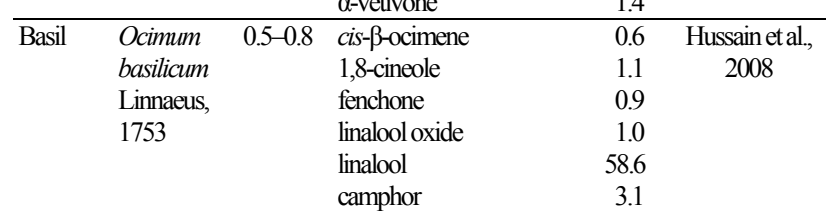

The third experiment was performed in a container $(50 \times 33 \times 19 \mathrm{~cm})$ in which wheat flour of highest sort was put $(400 \mathrm{~g})$ in a $1 \mathrm{~cm}$ layer. Then, 17 plastic cups with removed bottom ( $100 \mathrm{~mL}$ capacity) were put in the container at a distance of $0.5 \mathrm{~cm}$ one from another with $80 \mathrm{~g}$ of flour and 5 cups with 5 larvae of T. molitor in each. In 15 cups, into flour, a cotton disk of $0.4 \mathrm{~cm}$ diameter, saturated with $0.06 \mathrm{~mL}$ of essential oil of one of the plants $\left(0.48 \mathrm{~mL} / \mathrm{cm}^{2}\right)$, was placed at a $3 \mathrm{~cm}$ depth. For each of the 20 studied types of essential oils (Table 3) we used one cup. The other two cups were the control ( a $4 \mathrm{~cm}$ diameter cotton disk not processed with any of the essential oils was placed in them). Each cup was covered with a separate plastic cover to prevent mixing of the odours of the essential oils. The experiment was performed in five replications $(n=5)$. Duration of each experiment was 48 hours. After this period, the flour from the cups was sieved for counting live and dead insects. The results of the second and the third experiments were statistically analyzed in Statistica 8.0 (Statsoft Inc., USA) software pack. The differences between the selections were considered reliable at $\mathrm{P}<0.05$ (one-way ANOVA).

Table 3

Essential oils used in the experiment on determining migratory activity of Tenebrio molitor

\begin{tabular}{|c|c|c|c|c|c|}
\hline \multirow{2}{*}{$\begin{array}{l}\text { Sub- } \\
\text { stance }\end{array}$} & \multirow[b]{2}{*}{ Plant } & \multicolumn{2}{|c|}{ Chemical composition } & \multirow[b]{2}{*}{ ISO } & \multirow{2}{*}{$\begin{array}{l}\text { Refe- } \\
\text { rences }\end{array}$} \\
\hline & & compounds & $\begin{array}{c}\text { concen- } \\
\text { tration, } \%\end{array}$ & & \\
\hline Tea & Melaleuca & $\alpha$-pinene & 2.1 & 4730 & Cox \\
\hline tree & alternifolia & $\alpha$-terpinene & 8.3 & & etal., \\
\hline \multirow[t]{5}{*}{ oil } & (Maiden \& & $p$-cymene & 2.3 & & 2001 \\
\hline & Betche) & limonene & 1.1 & & \\
\hline & Cheel, & 1,8-cineole & 4.5 & & \\
\hline & 1925 & $\gamma$-terpinene & 17.8 & & \\
\hline & & $\alpha$-terpinolene & 3.3 & & \\
\hline
\end{tabular}




\begin{tabular}{|c|c|c|c|c|c|c|c|c|c|c|c|}
\hline \multirow{2}{*}{$\begin{array}{c}\text { Sub- } \\
\text { stance }\end{array}$} & \multirow[b]{2}{*}{ Plant } & \multicolumn{2}{|c|}{ Chemical composition } & \multirow[b]{2}{*}{ ISO } & \multirow{2}{*}{$\begin{array}{l}\text { Refe- } \\
\text { rences }\end{array}$} & & & Chemical col & & & \\
\hline & & compounds & $\begin{array}{l}\text { concen- } \\
\text { tration, } \%\end{array}$ & & & stance & Plant & compounds & $\begin{array}{l}\text { concen- } \\
\text { tration, } \%\end{array}$ & ISO & rences \\
\hline & & terpinen-4-ol & 39.8 & & & & & germacrene D & 2.10 & & \\
\hline & & $\alpha$-terpineol & 3.4 & & & & & viridiflorene & 3.29 & & \\
\hline & & aromadendrene & 1.2 & & & & & $\alpha$-muurolene & 2.70 & & \\
\hline & & viridiforene & 1.2 & & & & & $\gamma$-cadinene & 1.57 & & \\
\hline & & $\delta$-cadinene & 1.5 & & & & & $\delta$-cadinene & 5.97 & & \\
\hline Euca- & Eucalyptus & a-pinene & 5.65 & 770 & Abdossi & & & ledol & 1.29 & & \\
\hline lyptus & globulus & $\beta$-pinene & 0.31 & & etal., & & & spathulenol & 2.02 & & \\
\hline oil & Labillar- & sabinene & 0.65 & & 2015 & & & globulol & 1.67 & & \\
\hline & dière, 1861 & limonene & 0.84 & & & & & $\beta$-bisabolol & 1.26 & & \\
\hline & & 1,8-cineole & 76.65 & & & & & tetradecanol & 4.27 & & \\
\hline & & $\gamma$-terpinene & 0.63 & & & & & epi- $\alpha$-bisabolol & 2.08 & & \\
\hline & & terpien-4-ol & 0.37 & & & $\begin{array}{l}\text { Juniper } \\
\text {. }\end{array}$ & Juniperus & $\alpha$-thujene & 1.68 & 8897 & Chat- \\
\hline & & $\alpha$-terpineol & 1.96 & & & & communis & $\alpha$-pinene & 41.25 & & zopou- \\
\hline & & $\alpha$-terpineol acetate & 4.85 & & & & Linnaeus, & sabinene & 17.38 & & lou \& \\
\hline & & isoledene & 0.54 & & & & 1753 & $\beta$-pinene & 2.05 & & Katsio- \\
\hline & & $\alpha$-gurjunene & 0.85 & & & & & myrcene & 2.66 & & tis, 1993 \\
\hline & & $\beta$-gurjunene & 0.36 & & & & & $\alpha$-terpinene & 1.22 & & \\
\hline & & alloaromadendrene & 3.98 & & & & & limonene & 4.23 & & \\
\hline & & aromadendrene & 0.51 & & & & & 1,8-cineole & 1.21 & & \\
\hline Laven- & Lavandula & camphene & 1.37 & 3515 & Jianu & & & $\gamma$-terpinene & 2.09 & & \\
\hline deroil & angusti- & $\beta$-myrcene & 2.03 & & etal., & & & terpinolene & 1.16 & & \\
\hline & folia & D-limonene & 2.10 & & 2013 & & & terpinen-4-ol & 2.78 & & \\
\hline & Miller, & $\beta$-phellandrene & 16.00 & & & & & $\beta$-caryophyllene & 1.69 & & \\
\hline & 1768 & 1,8-cineole & 15.69 & & & & & $\alpha$-humulene & 1.56 & & \\
\hline & & terpinen-4ol & 9.57 & & & & & germacrene D & 1.83 & & \\
\hline & & borneol & 5.07 & & & Ginger & Zingiber & camphene & 3.0 & 16928 & Singh \\
\hline & & $\alpha$-terpineol & 6.00 & & & & officinale & $\beta$-phellandrene & 1.4 & & etal., \\
\hline & & santalene & 4.50 & & & & Roscoe, & 1,8-cineole & 1.9 & & 2008 \\
\hline & & caryophyllene & 24.12 & & & & 1807 & borneol & 2.1 & & \\
\hline Orange & Citrus & $\alpha$-pinene & 0.36 & 3140 & Singh & & & neral & 7.4 & & \\
\hline oil & sinensis & sabinine & 0.37 & & etal., & & & geraniol & 3.4 & & \\
\hline & (Linnaeus) & $\beta$-myrcene & 1.71 & & 2010 & & & geranial & 25.9 & & \\
\hline & Osbeck & octanal & 0.43 & & & & & ar-curcumene & 6.6 & & \\
\hline & (pro.sp.) & limonene & 90.66 & & & & & $\alpha$-zingiberene & 9.5 & & \\
\hline & & linalylacetate & 2.80 & & & & & (E,E)- $\alpha$-farmesene & 7.6 & & \\
\hline & & t-sabinine hydrate & 0.42 & & & & & $\beta$-sesquiphellandrene & 5.1 & & \\
\hline & & laevo- $\beta$-pinene & 0.46 & & & & & trans-nerolidol & 1.5 & & \\
\hline & & geranyl formate & 0.65 & & & & & zingiberenol & 1.7 & & \\
\hline Grape- & Citrus & $\alpha$-pinene & 0.4 & 3053 & Uysal & & & $\beta$-eudesmol & 1.0 & & \\
\hline fruit oil & paradisi & sabinene & 0.3 & & etal., & Cedar & Cedrus & $\alpha$-terpinene & 1.02 & 9843 & Derwich \\
\hline & Macfa- & $\beta$-pinene & 0.8 & & 2011 & oil & atlantica & cis-ocimene & 1.62 & & etal., \\
\hline & dyen, 1830 & $\beta$-myrcene & 0.7 & & & & (Endlicher) & humulene & 1.30 & & 2010 \\
\hline & & $\alpha$-terpinene & 0.7 & & & & G. Manetti & $\beta$-caryophyllene & 3.14 & & \\
\hline & & limonene & 91.5 & & & & ex Car- & $\sigma$-himachalene & 7.62 & & \\
\hline & & linalool & 1.1 & & & & rière, 1855 & cis- $\alpha$-atlantone & 6.78 & & \\
\hline & & trans-limonene oxide & 0.9 & & & & & himachalol & 5.26 & & \\
\hline & & citronellal & 0.4 & & & & & $\alpha$-himachalene & 4.15 & & \\
\hline & & $\alpha$-terpineol & 0.3 & & & & & $\alpha$-pinene & 14.85 & & \\
\hline & & nerol & 0.3 & & & & & $\beta$-pinene & 1.35 & & \\
\hline & & neral & 0.4 & & & & & himachalene & 10.14 & & \\
\hline & & geraniol & 0.3 & & & & & cadinene & 3.02 & & \\
\hline & & geranial & 0.4 & & & & & isocaryophillene & 1.10 & & \\
\hline Rose- & Rosmari- & $\alpha$-pinene & 14.90 & 1342 & Gachkar & & & $\beta$-himachalene & 9.89 & & \\
\hline mary oil & & camphene & 3.33 & & etal., & & & germacrene-D & 3.52 & & \\
\hline & officinalis & $\beta$-octanone & 1.61 & & 2007 & & & $\beta$-copaene & 2.26 & & \\
\hline & Linnaeus, & myrcene & 2.07 & & & & & cymene & 1.05 & & \\
\hline & 1753 & 1,8-cineole & 7.43 & & & & & 3-carene & 1.10 & & \\
\hline & & linalool & 14.90 & & & & & verbenol & 2.24 & & \\
\hline & & camphor & 4.97 & & & & & limonene & 2.01 & & \\
\hline & & borneol & 3.68 & & & & & ylangene & 2.20 & & \\
\hline & & terpinen-4-ol & 1.70 & & & & & $\beta$-phellandrene & 2.19 & & \\
\hline & & verbinone & 1.94 & & & & & $\gamma$-amorphane & 2.22 & & \\
\hline & & piperitone & 23.70 & & & Spruce & Picea & santene & 2.27 & - & Radules- \\
\hline & & bornyl acetate & 3.08 & & & & abies & $\alpha$-pinene & 5.40 & & cu et al., \\
\hline & & $\beta$-caryophyllene & 2.68 & & & & (Linnaeus) & camphene & 7.55 & & 2011 \\
\hline & & cis- $\beta$-farmesene & 1.26 & & & & H. Kars- & limonene & 9.29 & & \\
\hline & & $\alpha$-bisabolol & 1.01 & & & & ten., 1881 & bomeol & 1.11 & & \\
\hline Cin- & Cinna- & heptanal & 1.09 & - & Jayapra- & & & bornyl acetate & 11.78 & & \\
\hline namon- & moтum & nonanal & 1.09 & & kasha & & & $\alpha$-muurolene & 1.61 & & \\
\hline oil & verum & $\alpha$-copaene & 23.05 & & etal., & & & $\gamma$-cadinene & 1.54 & & \\
\hline & J.Presl, & $\alpha$-bergamotene & 27.38 & & 2002 & & & $\delta$-cadinene & 9.49 & & \\
\hline & 1825 & trans-cinnamyl acetate & 2.41 & & & & & nerolidol & 1.01 & & \\
\hline & & aromadendrene & 1.79 & & & & & $\alpha$-muurolol & 11.01 & & \\
\hline & & $\alpha$-humulene & 6.19 & & & & & $\delta$-cadinol & 1.48 & & \\
\hline
\end{tabular}




\begin{tabular}{|c|c|c|c|c|c|}
\hline \multirow{2}{*}{$\begin{array}{l}\text { Sub- } \\
\text { stance }\end{array}$} & \multirow[b]{2}{*}{ Plant } & \multicolumn{2}{|l|}{ Chemical composition } & \multirow[b]{2}{*}{ ISO } & \multirow[b]{2}{*}{$\begin{array}{l}\text { Refe- } \\
\text { rences }\end{array}$} \\
\hline & & compounds & $\begin{array}{c}\text { concen- } \\
\text { tration, \% }\end{array}$ & & \\
\hline & & $\alpha$-cadinol & 21.39 & & \\
\hline & & manool & 3.58 & & \\
\hline \multirow{25}{*}{$\begin{array}{l}\text { Thuja } \\
\text { oil }\end{array}$} & \multirow{25}{*}{$\begin{array}{l}\text { Thuja } \\
\text { occiden- } \\
\text { talis } \\
\text { Linnaeus, } \\
1753\end{array}$} & $\alpha$-thujene & 1.46 & \multirow[t]{25}{*}{-} & \multirow{25}{*}{$\begin{array}{c}\text { Jirovetz } \\
\text { et al., } \\
2006\end{array}$} \\
\hline & & $\alpha$-pinene & 3.33 & & \\
\hline & & camphene & 2.55 & & \\
\hline & & $\alpha$-fenchene & 2.04 & & \\
\hline & & sabinene & 12.14 & & \\
\hline & & $\beta$-pinene & 1.14 & & \\
\hline & & myrcene & 4.05 & & \\
\hline & & $p$-cymene & 2.37 & & \\
\hline & & $\alpha$-terpinene & 1.83 & & \\
\hline & & limonene & 2.36 & & \\
\hline & & $\beta$-phellandrene & 1.65 & & \\
\hline & & $\gamma$-terpinene & 2.29 & & \\
\hline & & trans-sabinene hydrate & 1.09 & & \\
\hline & & terpinolene & 2.32 & & \\
\hline & & fenchone & 12.87 & & \\
\hline & & linalool & 1.89 & & \\
\hline & & $\alpha$-thujone & 2.76 & & \\
\hline & & $\beta$-thujone & 9.48 & & \\
\hline & & camphor & 1.24 & & \\
\hline & & terpinen-4ol & 3.32 & & \\
\hline & & linalyl acetate & 1.24 & & \\
\hline & & sabinyl acetate & 16.55 & & \\
\hline & & terpinyl acetate & 1.17 & & \\
\hline & & $\beta$-caryophyllene & 1.23 & & \\
\hline & & $\delta$-cadinene & 1.29 & & \\
\hline Geraniu & Pelargo- & linalool & 5.60 & 4731 & Bouk- \\
\hline moil & nium & rose oxide-trans & 2.01 & & hris \\
\hline & graveolens & iso-menthone & 4.42 & & etal., \\
\hline & L'Héritier, & $\beta$-citronellol & 21.93 & & 2012 \\
\hline & 1789 & geraniol & 11.07 & & \\
\hline & & citronellyl formate & 13.24 & & \\
\hline & & geranyl formate & 6.22 & & \\
\hline & & $\beta$-bourbonene & 3.14 & & \\
\hline & & trans-caryophyllene & 1.02 & & \\
\hline & & germacrene D & 4.33 & & \\
\hline & & viridiflore & 2.35 & & \\
\hline & & $\delta$-cadinene & 2.38 & & \\
\hline & & $\delta$-cadinene & 1.33 & & \\
\hline & & $\alpha$-agarofuran & 1.28 & & \\
\hline & & 10-epi- $\gamma$-eudesmol & 7.92 & & \\
\hline & & geranyl tiglate & 2.39 & & \\
\hline Sandal- & Ptero- & cis-a-santalol & 31.67 & 3518 & Suba- \\
\hline wood & carpus & epi-a-bisabalol & 1.44 & & singhe \\
\hline oil & santalinus & epi- $\beta$-santalol & 2.36 & & etal., \\
\hline & Linnaeus & cis- $\beta$-santalol & 14.50 & & 2013 \\
\hline & filius, 1782 & cis-nuciferol & 1.02 & & \\
\hline & & $\gamma$-curcumen-12-ol & 1.68 & & \\
\hline & & $\beta$-curcumen-12-ol & 2.35 & & \\
\hline Lime & Citrus & 2,3-dimethyl-2,3-butanediol & 1.67 & - & Sandoval- \\
\hline oil & aurantii- & resorcinol & 3.65 & & Monte- \\
\hline & folia & 1-methoxycyclohexene & 8.00 & & mayor \\
\hline & (Christ- & linalool oxide & 1.18 & & etal., \\
\hline & mann) & corylone & 6.93 & & 2012 \\
\hline & Swingle, & terpinen-4ol & 1.66 & & \\
\hline & 1913 & $\alpha$-terpineol & 5.97 & & \\
\hline & & 3-methyl-1,2-cyclopentanedione & 8.27 & & \\
\hline & & 3,7-dimethyl-(z)-2,6-octadienal & 1.09 & & \\
\hline & & geraniol & 1.15 & & \\
\hline & & citral & 2.21 & & \\
\hline & & 7-methyl-(Z)-8-tetradecen-1-ol acetate & 2.83 & & \\
\hline & & geranyl acetone & 1.84 & & \\
\hline & & bergamotene & 1.00 & & \\
\hline & & (z)-8-methyl-9-tetradecenoic acid & 1.24 & & \\
\hline & & trans- $\alpha$-bisabolene & 1.02 & & \\
\hline & & caryophyllene oxide & 3.02 & & \\
\hline & & spathulenol & 1.95 & & \\
\hline & & umbelliferone & 4.36 & & \\
\hline & & palmitic acid & 6.89 & & \\
\hline & & 5,7-dimethoxycoumarin & 15.80 & & \\
\hline & & 5-methoxypsoralen & 1.14 & & \\
\hline & & 5,8-dimethoxypsoralen & 6.08 & & \\
\hline
\end{tabular}

Note: ${ }^{*}$-number of ISO standard.
All the variants of the experiment were performed in the same conditions. Fluctuations in temperature over the day did not exceed $3{ }^{\circ} \mathrm{C}$ $\left(+18 \ldots+21{ }^{\circ} \mathrm{C}\right)$, length of daylight in October-November of 2018 equalled $8.30-11.00 \mathrm{~h}$ and was prolonged by artificial illumination to 14 hours; relative air moisture was $60-70 \%$.

\section{Results}

The impact of essential oils on movement of $S$. granaries in conditions of a $48 \mathrm{~h}$ laboratory experiment is demonstrated in Figures 2-8. Essential oils from $C$. sinensis and $P$. abies stimulated migratory activity of $S$. granarius and are promising for use as repellents.

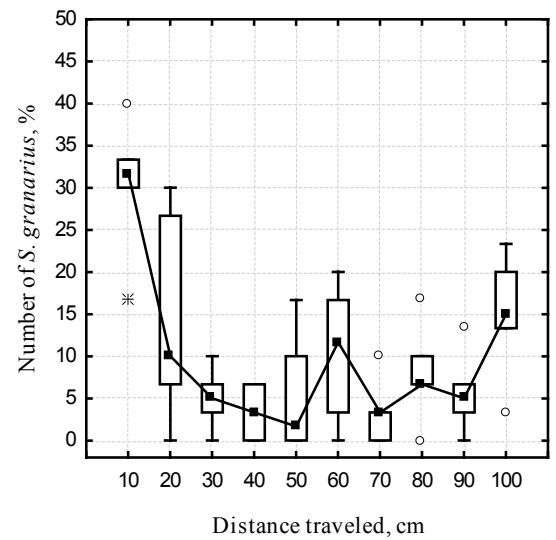

Fig. 1. Migration of S. granarius in pure fodder substrate over 48 hour laboratory experiment

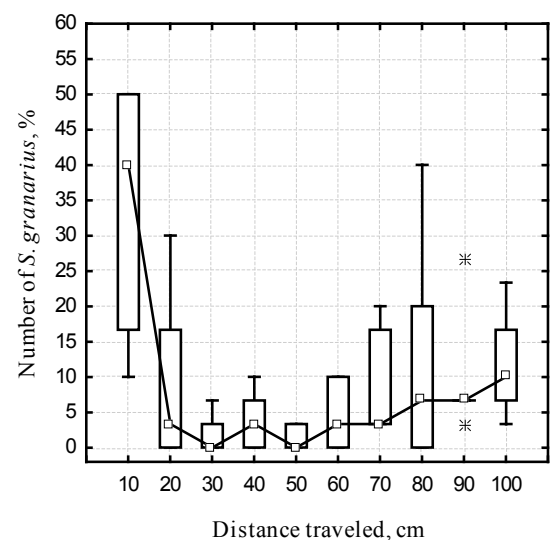

Fig. 2. Impact of essential oil of $M$. alternifolia on migratory activity of $S$. granarius: for 5 experiments $\chi^{2}=0.427(\mathrm{P}=0.999)$

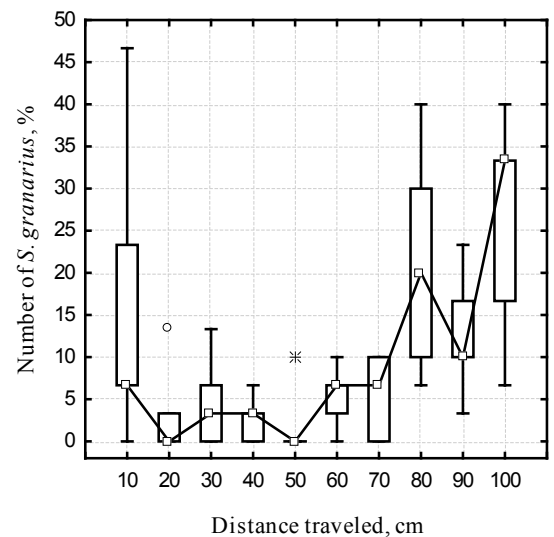

Fig. 3. Impact of essential oil from E. globulus on migratory activity of $S$. granarius: for 5 experiments $\chi^{2}=0.452(\mathrm{P}=0.999)$

The influence of dry plants of movement of larvae of $T$. molitor in the laboratory experiment which lasted 48 hours is demonstrated in Table 4. 
The strongest effect on movement activity of $T$. molitor larvae in the fodder substrate was shown by $O$. vulgare and $E$. globosus $(\mathrm{P}<0.01)$. Both plants exert strong repellent action against larvae of $T$. molitor.

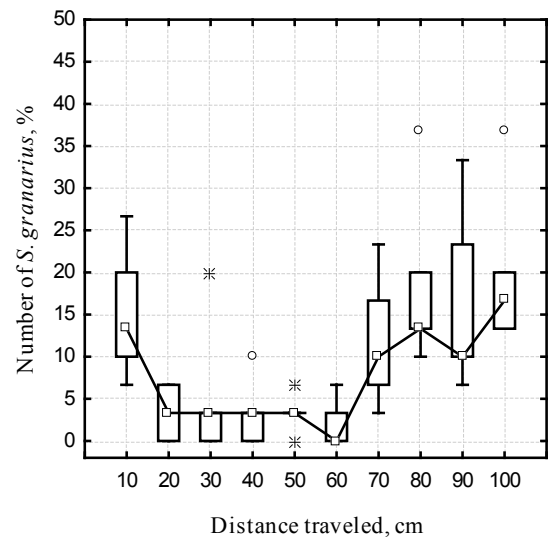

Fig. 4. Impact of essential oil from L. angustifolia on migratory activity of S. granarius: for 5 experiment $\chi^{2}=1.524(\mathrm{P}=0.997)$

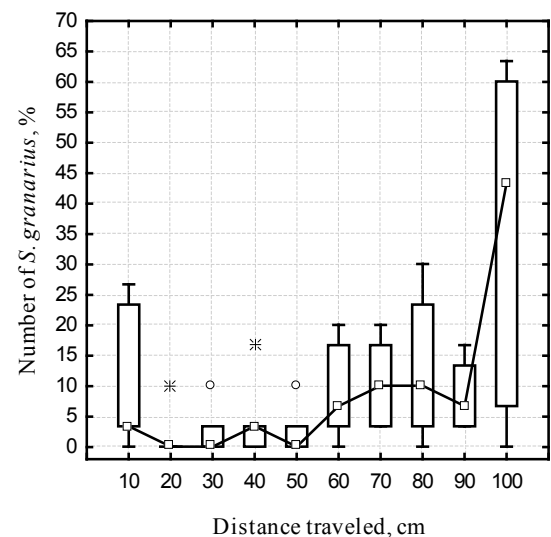

Fig. 5. Impact of essential oil from $M$. officinalis on migratory activity of $S$. granarius: for 5 experiments $\chi^{2}=2.614(\mathrm{P}=0.978)$

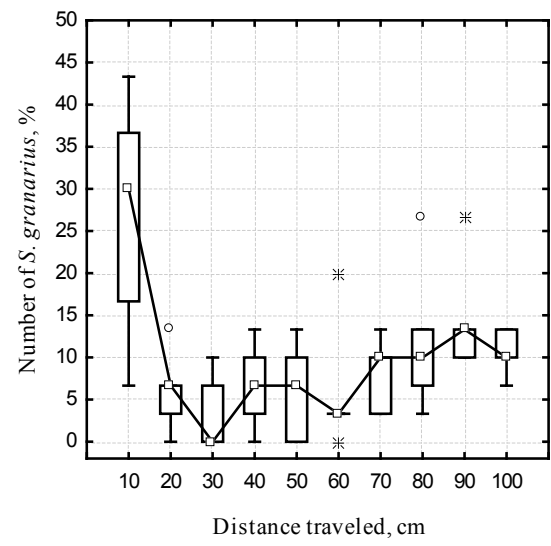

Fig. 6. Impact of essential oil from $C$. bergamia on migratory activity of $S$. granarius: for 5 experiments $\chi^{2}=0.900(\mathrm{P}=0.999)$

Impact of essential oils on movement of imagoes of T. molitor in the $48 \mathrm{~h}$ laboratory experiment is demonstrated in Table 5 . The strongest effect on distribution of larvae of $T$. molitor in the fodder substrate was exerted by essential oils from $J$. communis, $P$. abies, $P$. santalinus, C. sinensis and $C$. aurantiifolia $(\mathrm{P}<0.01)$.

\section{Discussion}

The obtained data indicate that the essential oils from $C$. sinensis and $P$. abies exert notable repellent action towards $S$. granarius at concentration of $0.48 \mathrm{~mL} / \mathrm{cm}^{2}$. Repellent effect on $T$. molitor was displayed 366 by dried and cut leaves of $O$. vulgare and E. globulus, and also essential oils from J. communis, P. abies, P. santalinus, C. sinensis and C. aurantiifolia $(\mathrm{P}<0.01)$.

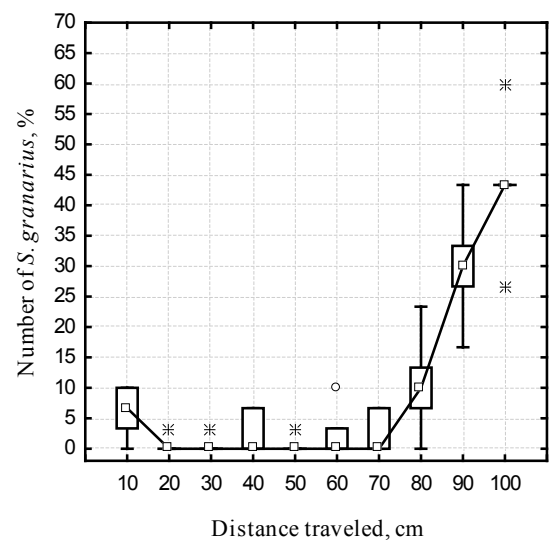

Fig. 7. Impact of essential oil from C. sinensis on migratory activity of S. granarius: for 5 experiments $\chi^{2}=1.604(\mathrm{P}=0.996)$

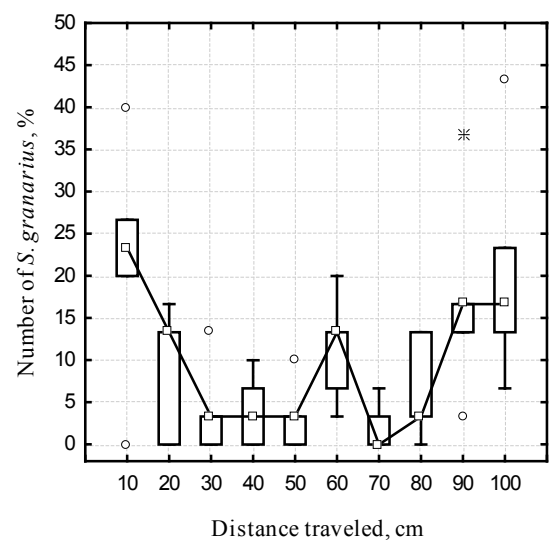

Fig. 8. Impact of essential oil from $C$. paradisi on migratory activity of $S$. granarius: for 5 experiments $\chi^{2}=0.598(\mathrm{P}=0.999)$

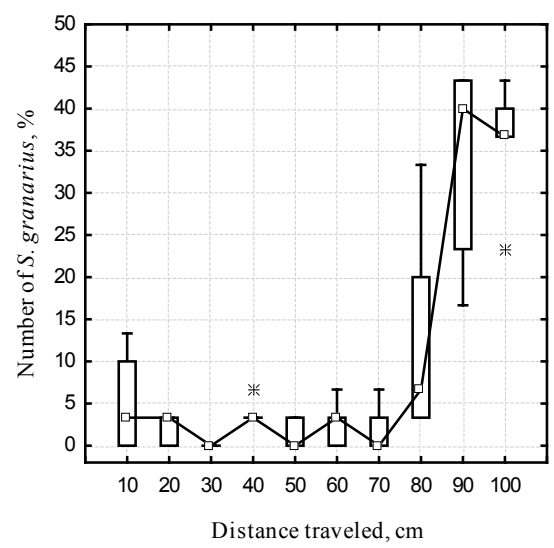

Fig. 9. Impact of essential oil from $P$. abies on migratory activity of $S$. granarius: for 5 experiments $\chi^{2}=3.202(\mathrm{P}=0.956)$

Absence of notable effects among the rest of the tested samples can be related to insufficient concentration of essential oils, or resistance of the pest species. Resistance of insects to the vapours of essential oils can be associated with the activity of cytochromes of $\mathrm{P}_{450}$-dependent monooxygenase, carboxyl esterase, superoxide dismutase and catalase (Ryan \& Byrne, 1988; Boyer et al., 2012).

Today, the effect of essential oils and their constituents on S. granarius and T. molitor and other economically harmful species is described in a number a scientific works. Yildrim et al. (2005) studied efficiency of eight essential oils: Hypericum scabrum Linnaeus, 1753, Hyssopus officinalis Linnaeus, (1753), Micromeria fruticosa Druce, 1914, Origa- 
num acutidens (Handel-Mazzetti) Ietswaart, Satureja hortensis Linnaeus, 1753, Salvia limbata C. A. Meyer, 1831 and S. nemorosa Linnaeus, 1762 against $S$. granarius. Mortality of insects at concentration of essential oils equaling $10 \mu \mathrm{L}$ was $74 \%, 66 \%, 73 \%, 4 \%, 12 \%, 10 \%$ and $14 \%$, respectively. Level of mortality rose with increase in the concentration of essential oils and duration of their action.

\section{Table 4}

Impact of dry medical plants ( $40 \mathrm{~g}$ of dry leaves per $1 \mathrm{~kg}$ of wheat grain) on distribution of larvae of Tenebrio molitor in fodder substrate in conditions of laboratory experiment

\begin{tabular}{|c|c|c|c|c|c|c|}
\hline \multirow[t]{2}{*}{ Species of plant } & \multicolumn{2}{|c|}{$\begin{array}{l}\text { Plant with addition of dry leaves } \\
\text { in grain, } n=50\end{array}$} & \multicolumn{2}{|c|}{$\begin{array}{c}\text { Section without addition } \\
\text { of dry leaves to the grain, } n=50\end{array}$} & \multirow{2}{*}{$\begin{array}{c}\mathrm{F} \\
\left(\mathrm{F}_{0.05}=9.42\right)\end{array}$} & \multirow[t]{2}{*}{$\mathrm{P}$} \\
\hline & $\mathrm{x}, \% *$ & $\mathrm{SD}, \% *$ & $\mathrm{x}, \% *$ & $\mathrm{SD}, \% *$ & & \\
\hline Salvia officinalis & 101.1 & 26.0 & 98.9 & 22.8 & 0.22 & 0.642 \\
\hline Helichrysum arenarium & 99.0 & 17.7 & 101.3 & 19.3 & 0.39 & 0.536 \\
\hline Origanum vulgare & 87.8 & 18.5 & 111.8 & 19.3 & 40.33 & $6.7 \cdot 10^{-9}$ \\
\hline Rhododendron tomentosum & 99.1 & 23.5 & 100.9 & 22.5 & 0.16 & 0.689 \\
\hline Aristolochia clematitis & 99.6 & 19.0 & 100.6 & 17.9 & 0.07 & 0.787 \\
\hline Lavandula angustifolia & 102.0 & 24.3 & 98.1 & 18.3 & 0.84 & 0.362 \\
\hline Melissa officinalis & 99.2 & 22.6 & 100.6 & 19.7 & 0.11 & 0.742 \\
\hline Matricaria chamomilla & 103.4 & 30.8 & 96.7 & 23.9 & 1.44 & 0.232 \\
\hline Ocimum basilicum & 99.6 & 25.3 & 100.3 & 19.3 & 0.02 & 0.885 \\
\hline Mentha piperita & 94.8 & 18.5 & 104.6 & 26.6 & 4.51 & 0.036 \\
\hline Eucalyptus globulus & 88.5 & 24.0 & 112.1 & 27.3 & 21.10 & $1.3 \cdot 10^{-5}$ \\
\hline Artemisia absinthium & 98.5 & 23.3 & 101.8 & 22.6 & 0.50 & 0.480 \\
\hline Laurus nobilis & 104.6 & 28.2 & 95.0 & 21.8 & 3.58 & 0.061 \\
\hline Thymus serpyllum & 100.6 & 27.5 & 99.4 & 27.1 & 0.05 & 0.827 \\
\hline Rosmarinus officinalis & 97.7 & 32.2 & 102.0 & 21.3 & 0.60 & 0.441 \\
\hline Syzygium aromaticum & 99.9 & 23.6 & 100.1 & 23.6 & 0.01 & 0.962 \\
\hline Achillea millefolium & 100.9 & 28.8 & 98.9 & 26.8 & 0.13 & 0.719 \\
\hline Tanacetum vulgare & 97.9 & 24.6 & 102.4 & 30.0 & 0.67 & 0.416 \\
\hline
\end{tabular}

Note: * $\mathrm{x}$ is share of initial number of larvae distributed in particular section of the tube (\%).

Table 5

Impact of essential oils on distribution of T. molitor larvae in fodder substrate in conditions of laboratory experiment

\begin{tabular}{lrccc}
\hline \multicolumn{1}{c}{ Essential oil } & $\mathrm{x}, \% *$ & $\mathrm{SD}, \% *$ & $\begin{array}{c}\mathrm{F} \\
\left(\mathrm{F}_{0.05}=10.83\right)\end{array}$ & $\mathrm{p}$ \\
\hline Control & 78.8 & 29.8 & - & - \\
Melaleuca alternifolia & 68.2 & 14.0 & 0.84 & 0.370 \\
Rosmarinus officinalis & 65.9 & 14.2 & 1.25 & 0.276 \\
Cinnamomum verum & 103.5 & 33.4 & 3.24 & 0.086 \\
Juniperus communis & 32.9 & 31.4 & 11.63 & $2.5 \cdot 10^{-3}$ \\
Zingiber officinale & 70.6 & 16.7 & 0.49 & 0.492 \\
Cedrus atlantica & 68.2 & 20.0 & 0.77 & 0.390 \\
Picea abies & 14.1 & 19.5 & 28.92 & $2.1 \cdot 10^{-5}$ \\
Thuja occidentalis & 84.7 & 14.2 & 0.26 & 0.617 \\
Pelargonium graveolens & 82.4 & 20.0 & 0.08 & 0.773 \\
Lavandula angustifolia & 110.6 & 23.5 & 6.51 & 0.018 \\
Eucalyptus globulus & 63.5 & 20.0 & 1.60 & 0.219 \\
Pterocarpus santalinus & 14.1 & 16.7 & 30.18 & $1.6 \cdot 10^{-5}$ \\
Citrus aurantiifolia & 40.0 & 25.5 & 9.36 & $5.7 \cdot 10^{-3}$ \\
Citrus sinensis & 16.5 & 21.2 & 26.11 & $4.0 \cdot 10^{-5}$ \\
Citrus paradisi & 87.1 & 26.5 & 0.41 & 0.527 \\
\hline
\end{tabular}

Note: $*-\mathrm{x}$ is share of the initial number of larvae placed in the cup before the beginning of the experiment $(\%)$.

Rozman et al. (2006) studied the efficacy of essential oils from L. angustifolia, L. nobilis, R. officinalis and Thymus vulgaris Linnaeus, 1753, and also their components: 1,8-cineole, camphor, eugenol, linalool, carvacrol, thymol, borneol, bornylacetate and lynalylacetate against $S$. granarius. The studied substances in concentration of $0.1 \mu \mathrm{L} / 720 \mathrm{~mL}$ demonstrated high efficiency with average mortality of $96.5-100.0 \%$ after $24 \mathrm{~h}$ exposure.

Ebadollah \& Mahboubi (2011) studied the efficacy of essential oil from Azilia eryngioides (Pau) Hedge \& Lamond, 1987 against S. granarius. The studied oil had high insecticidal activity, $100 \%$ death rate was achieved at impact of concentration of $37.0 \mu \mathrm{L} / \mathrm{L}$ over $28 \mathrm{~h}$. Values of $\mathrm{LC}_{50}$ of essential oil from A. eryngioides for $S$. granarius equaled $20.1 \mu \mathrm{L} / \mathrm{L}$ after $24 \mathrm{~h}$ of impact.

In a study on toxicity of different essential oils against $S$. granarius, Lamiri et al. (2001) determined that the highest toxic effect on the insect was caused by essential oils from Mentha pulegium Linnaeus, 1753, M. spicata Linnaeus, 1753, E. globulus and Origanum compactum Bentham, which exerted killing effect on the insect in concentration of 10 and $5 \mu \mathrm{L} / \mathrm{mg}$ over 24 and $48 \mathrm{~h}$ respectively.
Mahmoudvand et al. (2011) studied toxicity of essential oil from C. sinensis for S. granarius, Tribolium castaneum (Herbst, 1797) and Callosobruchus maculatus (Fabricius, 1775). Values of $\mathrm{LC}_{50}$ of the tested substance equaled $367.8,391.3$ and $223.5 \mu \mathrm{L} / \mathrm{L}$ of air after $24 \mathrm{~h}$ and $320.5,362.4$ and 207.2 after $48 \mathrm{~h}$ of exposure for S. granarius, T. castaneum and C. maculatus, respectively. Mortality of the insects depended on concentration and duration of impact of the tested essential oil.

Di Stefano (2016) studied insecticidal activity of essential oil from L. angustifolia against $S$. granarius. Mortality of insects at concentration of essential oil equaling $449.1 \mu \mathrm{g} / \mathrm{mg}$ equaled $91.7 \%$ and $100.0 \%$ after 24 and $48 \mathrm{~h}$ of impact, respectively. Values of $\mathrm{LD}_{50}$ and $\mathrm{LD}_{90}$ equaled 83.8 and $379.7 \mu \mathrm{g} / \mathrm{mg}$ after $24 \mathrm{~h}$ and reduced to 58.3 and $208.3 \mu \mathrm{g} / \mathrm{mg}$ after $48 \mathrm{~h}$, respectively. Fumigant toxicity of essential oil from $L$. angustifolia for $S$. granarius reached its maximum at doses of 11.9 and $47.5 \mathrm{mg} / \mathrm{L}$ of air. Values of $\mathrm{LC}_{50}$ and $\mathrm{LC}_{90}$ equaled 1.6 and $4.1 \mathrm{mg} / \mathrm{L}$ at absence of substrate (grains of wheat) and 10.9 and $47.6 \mathrm{mg} / \mathrm{L}$ at presence of grains respectively. The studied essential oil had notable repellent activity against imagoes of $S$. granarius.

Study on toxicity of essential oils from Artemisia absinthium, A. santonicum Linnaeus, 1753 and $A$. spicigera $\mathrm{K}$. Koch, 1851, and also their constituents against $S$. granarius were undertaken by Kordali et al. (2006). Essential oils of all three species killed $80-90 \%$ of the insects at concentration of $9 \mathrm{~L} / \mathrm{L}$ of air during $48 \mathrm{~h}$ of exposure. Mortality increased with increase of doses of essential oils and duration of exposure. The main components of essential oils: borneol, bornyl acetate, camphor, $\alpha$-terpineol killed $100 \%$ of $S$. granarius at concentration equaling 1.0 L/L of air, and 1,8-cineole and terpinen-4-ol - at concentration of $0.5 \mathrm{~L} / \mathrm{L}$ of air, respectively, at over $12 \mathrm{~h}$ exposure.

Mohamed \& Abdelgaleil (2008) studied toxicity of different essential oils against Sitophilus oryzae (Linnaeus, 1763) and T. castaneum. Highest toxicity for $S$. oryzae was exhibited by essential oil from Citrus limon (Linnaeus) Osbeck, 1765, C. sinensis and $C$. paradisi, $\mathrm{LC}_{50}$ for which equaled 9.9, 19.7 and $24.1 \mathrm{mg} / \mathrm{L}$ of air, respectively. Highest toxicity against $T$. castaneum was exerted by $C$. sinensis and $C$. paradisi with parameters of $\mathrm{LC}_{50}$ equaling 24.6 and $25.5 \mathrm{mg} / \mathrm{L}$ of air, respectively.

Abdelgaleil et al. (2015) studied insecticidal activity of plant essential oils against $S$. oryzae. At fumigation, the most toxic for the insect were essential oils from $O$. vulgare, C. limon, Callistemon viminalis (Solander ex Gaertner) G. Done x Loudon (1830), Cupressus sempervirens Linnaeus, 1753 and $C$. sinensis, values of $\mathrm{LC}_{50}$ of which equaled $1.64,9.89,16.17,17.16$ and $19.65 \mathrm{mg} / \mathrm{L}$ of air, respectively. The highest contact toxicity towards $S$. oryzae was exhibited by essential oils from 
Artemisia judaica Linnaeus, $1759, C$. viminals and $O$. vulgare with parameters of $\mathrm{LC}_{50}$ equaling $0.08,0.09$ and $0.11 \mathrm{mg} / \mathrm{cm}_{2}$, respectively. Essential oil from A. judaica in concentration of $16.1 \mathrm{mg} / \mathrm{L}$ exerted inhibiting effect on the activity of acetylcholinesterase, whereas oils from $C$. viminals and $O$. vulgare were strong inhibitors of ATRs at concentration of 4.69 and $6.07 \mathrm{mg} / \mathrm{L}$, respectively. In a similar study, Lee et al. (2001) determined that most toxic essential oils for $S$. oryzae were those from E. globulus and R. officinalis, $\mathrm{LD}_{50}$ of which equaled 28.9 and $30.5 \mathrm{~L} / \mathrm{L}$ of air. Essential oils from L. angustifolia, T. vulgaris, Cananga odorata (Lamarck) Hooker \& Thomson, 1855 and C. paradisi were less toxic: $\mathrm{LD}_{50}-54.0,63.9,73.1$ and $87.0 \mathrm{~L} / \mathrm{L}$ of air, respectively. Most toxic for $S$. oryzae were the following components of essential oils: 1,8cineole, p-cymene, $\alpha$-pinene and limonene, $\mathrm{LD}_{50}$ of which equaled 23.5, 25.0, 54.9 and $61.5 \mathrm{~L} / \mathrm{L}$ of air, respectively.

AbdEl-Salam (2010) studied toxic action of essential oils for S. ory$z a e$. Percentage of mortality heightened with increase in concentration of different essential oils and period of exposure. Essential oils from C. verum and M. alternifolia killed $90.0 \%$ of insects at concentrations of 8.0 and $16.0 \mu \mathrm{L} / 50 \mathrm{~mL}$ of air, respectively at impact over 24 hours. Values of $\mathrm{LC}_{95}$ of essential oils from $C$. verum, S. aromaticum and $E$. globulus equaled 3.67, 4.07 and $8.73 \mu \mathrm{L} / 50 \mathrm{~mL}$ of air for $S$. oryzae at period of impact of 72 hours.

Insecticidal activity of essential oils against $S$. oryzae was studied by Yazdgerdian et al. (2015). The most active fumigants of the insect were Gaultheria procumbens Linnaeus, 1753, Thuja plicata Donn ex D. Don, 1824 and Bursera graveolens (Kunth) Triana \& Planchon, 1872, values of $\mathrm{LC}_{50}$ of which equaled $6.8,19.8$ and $21.4 \mu \mathrm{L} / \mathrm{L}$ of air, respectively.

Rajkumar et al. (2019) studied insecticidal activity of essential oil from M. piperita and its constituents against $S$. oryzae and $T$. castaneum. Values of $\mathrm{LC}_{50}$ of essential oil, menthone and menthol for S. oryzae equaled 43.2, 46.7 and $49.4 \mu \mathrm{L} / \mathrm{L}$ of air, and for $T$. castaneum $-48.7,51.9$ and $54.5 \mu \mathrm{L} / \mathrm{L}$ of air, respectively. Both insect species were observed to have dose-dependant inhibition of activity of acetylcholinesterase when exposed to essential oil from M. piperita, menthone and menthol concentration, $\mathrm{LC}_{50}$ equaled $29.7 \%, 18.8 \%$ and $14.3 \%$ for $S$. oryzae and $20.7 \%$, $13.7 \%$ and $9.2 \%$ for $T$. castaneum, respectively at 24 hours exposure. Also, under the impact of the studied substances in dose equal to $\mathrm{LC}_{50}$, an increase was observed in the activity of superoxide dismutase by 17.4, 15.2 and 13.3 units/mg of protein and 31.9, 28.3 and 25.3 units $/ \mathrm{mg}$ of protein for $S$. oryzae and $T$. castaneum, respectively.

Bertoli et al. (2012) studied biological activity of essential oils against Sitophilus zeamais (Motschulsky), 1855. Toxic effect of essential oils from A. millefolium and Foeniculum vulgare Miller, 1768 was displayed by minimum concentration of $0.05 \mu \mathrm{L}$, similar to the effect of $L$. angustifolia - at concentration of $0.10 \mu \mathrm{L}$. Level of mortality of insects was never higher than $76.0 \%$ (essential oil from L. angustifolia in dose of $0.75 \mu \mathrm{L}$ during more than 96 hours of exposure).

Buneri et al. (2019) studied efficiency of essential oil from Cedrus deodara (Roxburghe x D. Don) G. Don, 1830 in concentrations of $0.8 \%$, $1.5 \%, 3.0 \%, 6.0 \%$ and $12.0 \%$ as insecticide against larvae of $T$. molitor. Death rate of insects after 48 h equaled $17 \%, 33 \%, 49 \%, 64 \%$ and $87 \%$, respectively. $\mathrm{LC}_{50}$ of oil from $C$. deodara equaled $3.1 \%$. After the exposure, the insects were observed to have increase in the level of total protein from 2.12 to $4.14 \mathrm{mg} / \mathrm{mL}$. Essential oil from $C$. deodara has a notable repellent action against larvae of $T$. molitor.

Fazolin et al. (2007) studied toxicity of essential oils from Piper aduncum Linnaeus, 1753, P. hispidinervum C. de Candolle, 1914 and Tanaecium nocturnum (Barbosa Rodrigues) Bureau \& K. Schumann, 1896 against larvae of $T$. molitor. Values of $\mathrm{LC}_{50}$ for these essential oils were $0.045,0.033$ and $1.515 \mathrm{~mL} / \mathrm{cm}^{2}, \mathrm{LD}_{50}-0.000025,0,009$ and $0.000015 \mathrm{~mL} / \mathrm{g}$, respectively.

Study on repellent activity of essential oils from L. nobilis, C. bergamia, F. vulgare, Lavandula $\mathrm{x}$ intermedia Emeric ex Loiseleur-Deslongchamps in $0.01 \%$ and $0.10 \%$ concentrations against larvae of T. molitor was performed by Cosimi et al. (2009). Essential oil from $F$. vulgare had a repellent effect in concentrations of $0.10 \%$ after $1 \mathrm{~h}$ of exposure, whereas the rest of the oils showed low effectiveness even after $5 \mathrm{~h}$ of exposure. Wang et al. (2016) studied the effect of essential oils and their components on larvae of $T$. molitor. Significant repellent effect on the insect was presented by geraniol, cis-3-hexenol and ionone in concentration of $1 \mathrm{~mol} / \mathrm{L}$, and also essential oil of eucalypt in concentration of $1 \%$. Attractant effect on T. molitor was caused by isoeugenol and $\alpha$-pinene in concentrations of 1.0 and $0.1 \mathrm{~mol} / \mathrm{L}$, and also peppermint oil and turpentine oil in concentrations of $1.0 \%$ and $0.1 \%$.

Plata-Rueda et al. (2017) studied insecticidal activity of essential oil from Allium sativum Linnaeus, 1753 for different stages of T. molitor. The insect was more sensitive at the larva stage compared to the pupa and imago stages. Values of $\mathrm{LC}_{50}$ for the different stages equaled 0.771 , $2.371,2.032 \mu \mathrm{L} / \mathrm{mL}$, respectively at impact over $48 \mathrm{~h}$. Essential oil from A. sativum was noted for repellent action against $T$. molitor at all stages of the development, and also caused reduction of frequency of breathing of the insect after $1 \mathrm{~h}$ of impact.

George et al. (2009) studied toxicity of different essential oils for T. molitor. Most toxic for the insects were essential oils from Carum carvi Linnaeus, 1753, Mentha spicata Linnaeus, 1753 and J. communis, which killed $95 \%, 87 \%$ and $85 \%$, respectively at concentration of substances of $0.14 \mathrm{mg} / \mathrm{cm}^{3}$ after $24 \mathrm{~h}$ of impact. Less effective were oils from T. vulgaris and Piper nigrum Linnaeus, 1753, the killing power of which equaled $50 \%$ and $40 \%$, respectively at the same concentration and duration of exposure.

Kuusik et al. (1995) studied toxicity of extract of $R$. tomentosum for pupaes of T. molitor. Exposure of the pupae with extract of R. tomentosum led to different morphological effects depending on duration of exposure. The affected pupae transformed into immature imagoes. Furthermore, changes were observed in the movement activity of the pupae weak bending activity turned into energetic and more prolonged.

Effectiveness of plant essential oils against $T$. molitor was studied by Wang et al. (2015). Values of $\mathrm{LC}_{50}$ of essential oils from Litsea cubeba (Loureiro) Persoon, 1807 and C. limon equaled 19.6 and $42.2 \mathrm{mg} / \mathrm{cm}^{2}$ respectively at $24 \mathrm{~h}$ exposure and 13.9 and $21.2 \mathrm{mg} / \mathrm{cm}^{2}$ at $48 \mathrm{~h}$ exposure. The studied essential oils exhibited notable repellent activity against larvae of $T$. molitor, and also caused increase in duration of the development of larva stage.

Martínez et al. (2017) studied insecticidal activity of essential oils from $C$. verum and $S$. aromaticum, and also some of their components against $T$. molitor. Values of $\mathrm{LC}_{50}$ of essential oil from $C$. verum for larvae, pupae, and imagoes of T. molitor equaled 30.4, 10.7 and $29.8 \mu \mathrm{g} / \mathrm{mL}$, and $\mathrm{LC}_{50}$ of $S$. aromaticum were $35.1,6.5$ and $21.6 \mu \mathrm{g} / \mathrm{mL}$, respectively. $\mathrm{LC}_{50}$ of eugenol, caryophyllene oxide, $\alpha$-pinene $\alpha$-humulene and $\alpha$-phellandrene for larvae of T. molitor were 9.2, 9.2, 14.0, 15.2 and $17.1 \mu \mathrm{g} / \mathrm{mL}$, respectively. Caryophyllene oxide showed a notable repellent action towards larvae of T. molitor, while eugenol, $\alpha$-humulene and essential oil from $C$. verum showed an attractant effect.

Despite the relevance of developing methods of using essential oils and their constituents as insecticidal preparations, their introduction into technologies of integrated control of pests remains impossible due to insufficient amount of data on this problem, difficulties in standardization and control of plant production.

\section{Conclusions}

Notable repellent activity against $S$. granarius was exhibited by essential oils from C. sinensis and P. abies at concentration of $0.48 \mathrm{~mL} / \mathrm{cm}^{2}$. Repellent effect on T. molitor was displayed by dried and cut leaves of $O$. vulgare and E. globulus, and also essential oils from J. communis, $P$. abies, $P$. santalinus, $C$. sinensis and $C$. aurantiifolia $(\mathrm{P}<0.01)$. Therefore, out of 18 studied essential oils, only two samples had notable biological action on migratory ability of $S$. granarius and only five samples - on T. molitor. These data are confirmed by many studies on insecticidal activity of essential oils and the possibility of using them as ecologically safe natural pesticides. Studies on biological activity of essential oils against economically harmful species of insects is a relevant issue necessary for the development of ecologically-based control of agricultural pests.

\section{References}

Abd El-Salam, A. (2010). Fumigant toxicity of seven essential oils against the cowpea weevil, Callosobruchus maculatus (F.) and the rice weevil, Sitophi- 
lus oryzae (L.). Egyptian Academic Journal of Biological Sciences, F. Toxicology and Pest Control, 2(1), 1-6.

Abdelgaleil, S. A. M., Mohamed, M. I. E., Shawir, M. S., \& Abou-Taleb, H. K (2015). Chemical composition, insecticidal and biochemical effects of essential oils of different plant species from Northern Egypt on the rice weevil, $\mathrm{Si}$ tophilus oryzae L. Journal of Pest Science, 89(1), 219-229.

Abdossi, V., Moghaddam, E. Y., \& Hadipanah, A. (2015). Chemical composition of Eucalyptus globulus grown in Iran. Biological Forum, 7(2), 322-324.

Andoğan, B. C., Baydar, H., Kaya, S., Demirci, M., Özbașar, D., \& Mumcu, E. (2002). Antimicrobial activity and chemical composition of some essential oils. Archives of Pharmacal Research, 25(6), 860-864

Bakkali, F., Averbeck, S., Averbeck, D., \& Idaomar, M. (2008). Biological effects of essential oils - a review. Food and Chemical Toxicology, 46(2), 446-475.

Barnes, A. I., \& Siva-Jothy, M. T. (2000). Density-dependent prophylaxis in the mealworm beetle Tenebrio molitor L. (Coleoptera: Tenebrionidae): Cuticular melanization is an indicator of investment in immunity. Proceedings of the Royal Society of London, Series B: Biological Sciences, 267(1439), 177-182.

Benhalima, H., Chaudhry, M. Q., Mills, K. A., \& Price, N. R. (2004). Phosphine resistance in stored-product insects collected from various grain storage facilities in Morocco. Journal of Stored Products Research, 40(3), 241-249.

Bertoli, A., Conti, B., Mazzoni, V., Meini, L., \& Pistelli, L. (2012). Volatile chemical composition and bioactivity of six essential oils against the stored food insect Sitophilus zeamais Motsch. (Coleoptera, Dryophthoridae). Natural Product Research, 23(22), 2063-2071.

Boukhris, M., Simmonds, M. S. J., Sayadi, S., \& Bouaziz, M. (2012). Chemical composition and biological activities of polar extracts and essential oil of rose-scented geranium, Pelargonium graveolens. Phytotherapy Research, 27(8), 1206-1213.

Boyer, S., Zhang, H., \& Lempérière, G. (2012). A review of control methods and resistance mechanisms in stored-product insects. Bulletin of Entomological Research, 102(2), 213-229.

Boyko, A. A., \& Brygadyrenko, V. V. (2017). Changes in the viability of the eggs of Ascaris suum under the influence of flavourings and source materials approved for use in and on foods. Biosystems Diversity, 25(2), 162-166.

Buneri, I. D., Yousuf, M., Attaullah, M., Afridi, S., Anjum, S. I., Rana, H., Ansari, M. J. (2019). A comparative toxic effect of Cedrus deodara oil on larval protein contents and its behavioral effect on larvae of mealworm beetle (Tenebrio molitor) (Coleoptera: Tenebrionidae). Saudi Journal of Biological Sciences, 26(2), 281-285.

Burt, S. (2004). Essential oils: Their antibacterial properties and potential applications in foods - a review. International Journal of Food Microbiology, 94(3), 223-253.

Chalchat, J. C., Chabard, J. L., \& Gorunovic, M. S., Djermanovic, V., \& Bulatovic, V. (1995). Chemical composition of Eucalyptus globulus oils from the Montenegro coast and east coast of Spain. Journal of Essential Oil Research, $7(2), 147-152$.

Chatzopoulou, P. S., \& Katsiotis, S. T. (1993). Chemical investigation of the leaf oil of Juniperus communis L. Journal of Essential Oil Research, 5(6), 603-607.

Cosimi, S., Rossi, E., Cioni, P. L., \& Canale, A. (2009). Bioactivity and qualitative analysis of some essential oils from Mediterranean plants against storedproduct pests: Evaluation of repellency against Sitophilus zeamais Motschulsky, Cryptolestes ferrugineus (Stephens) and Tenebrio molitor (L.) Journal of Stored Products Research, 45(2), 125132

Costa, R., Dugo, P., Navarra, M., Raymo, V., Dugo, G., \& Mondello, L. (2010). Study on the chemical composition variability of some processed bergamot (Citrus bergamia) essential oils. Flavour and Fragrance Journal, 25(1), 4-12.

Cox, S. D., Mann, C. M., \& Markham, J. L. (2001). Interactions between components of the essential oil of Melaleuca alternifolia. Journal of Applied Microbiology, 91(3), 492-497.

Czinner, E., Lemberkovics, É., Bihátsi-Karsai, E., Vitányi, G., \& Lelik, L. (2000) Composition of the essential oil from the inflorescence of Helichrysum arenarium (L.) Moench. Journal of Essential Oil Research, 12(6), 728-730.

Dadalioğlu, I., \& Evrendilek, G. A. (2004). Chemical compositions and antibacterial effects of essential oils of Turkish oregano (Origanum minutiflorum), bay laurel (Laurus nobilis), Spanish lavender (Lavandula stoechas), and fennel (Foeniculum vulgare) on common foodborne pathogens. Journal of Agricultural and Food Chemistry, 52(26), 8255-8260.

Derwich, E., Benziane, Z., \& Boukir, A. (2009). Chemical composition and antibacterial activity of leaves essential oil of Laurus nobilis from Morocco. Australian Journal of Basic and Applied Sciences, 3(4), 3818-3824

Derwich, E., Benziane, Z., \& Boukir, A. (2010). Chemical composition and in vitro antibacterial activity of the essential oil of Cedrus atlantica. International Journal of Agriculture and Biology, 12, 381-385.

Dhouioui, M., Boulila, A., Chaabane, H., Zina, M. S., \& Casabianca, H. (2016). Seasonal changes in essential oil composition of Aristolochia longa L. ssp. paucinervis Batt. (Aristolochiaceae) roots and its antimicrobial activity. Industrial Crops and Products, 83, 301-306.
Di Stefano, M. G. (2016). Toxic, repellent and antifeedant activities of Lavandula angustifolia Miller (Lamiaceae) essential oil against Sitophilus granarius (L.) (Coleoptera, Curculionidae) adults. Universita Degli Studi Del Molisw, Campobasso.

Dunkel, F. V. (1992). The stored grain ecosystem: A global perspective. Journal of Stored Products Research, 28(2), 73-87.

Ebadollah, A., \& Mahboubi, M. (2011). Insecticidal activity of the essential oil isolated from Azilia eryngioides (Pau) Hedge et Lamond against two beetle pests. Chilean Journal of Agricultural Research, 71(3), 406-411.

Ebadollahi, A., \& Jalali Sendi, J. (2015). A review on recent research results on bio-effects of plant essential oils against major coleopteran insect pests. Toxin Reviews, 34(2), 76-91.

Fazolin, M., Estrela, J. L. V., Catani, V., Alécio, M. R., \& Lima, M. S. (2007) Propriedade inseticida dos óleos essenciais de Piper hispidinervum C. DC Piper aduncum L. e Tanaecium nocturnum (Barb. Rodr.) Bur. \& K. Shum sobre Tenebrio molitor L., 1758. Ciência e Agrotecnologia, 31(1), 113-120.

Flinn, P. W., Hagstrum, D. W., Reed, C., \& Phillips, T. W. (2003). United States Department of Agriculture-Agricultural Research Service stored-grain area wide integrated pest management program. Pest Management Science, 59(6-7), 614-618.

Gachkar, L., Yadegari, D., Rezaei, M., Taghizadeh, M., Astaneh, S., \& Rasooli, I (2007). Chemical and biological characteristics of Cuminum cyminum and Rosmarinus officinalis essential oils. Food Chemistry, 102(3), 898-904

George, D. R., Sparagano, O. A. E., Port, G., Okello, E., Shiel, R. S., \& Guy, J. H. (2009). Repellence of plant essential oils to Dermanyssus gallinae and toxicity to the non-target invertebrate Tenebrio molitor. Veterinary Parasitology, $162,129-134$

Grdiša, M., \& Gršić, K. (2013). Botanical insecticides in plant protection. Agriculturae Conspectus Scientificus, 78(2), 85-93.

Gutiérrez, M. M., Werdin-González, J. O., Stefanazzi, N., Bras, C., \& Ferrero, A. A. (2015). The potential application of plant essential oils to control Pediculus humanus capitis (Anoplura: Pediculidae). Parasitology Research, $115(2), 633-641$

Heuskin, S., Godin, B., Leroy, P., Capella, Q., Wathelet, J.-P., Verheggen, F., \& Lognay, G. (2009). Fast gas chromatography characterisation of purified semiochemicals from essential oils of Matricaria chamomilla L. (Asteraceae) and Nepeta cataria L. (Lamiaceae). Journal of Chromatography A, 1216(14), 2768-2775

Hussain, A. I., Anwar, F., Hussain Sherazi, S. T., \& Przybylski, R. (2008). Chemical composition, antioxidant and antimicrobial activities of basil (Ocimum basilicum) essential oils depends on seasonal variations. Food Chemistry, 108(3), 986-995.

Isman, M. B. (1997). Neem and other botanical insecticides: Barriers to commercialization. Phytoparasitica, 25(4), 339-344.

Isman, M. B. (2000). Plant essential oils for pest and disease management. Crop Protection, 19, 603-608.

Isman, M. B. (2004). Plant essential oils as green pesticides for pest and disease management. Agricultural Applications in Green Chemistry, 4, 41-51.

Isman, M. B. (2006). Botanical insecticides, deterrents, and repellents in modern agriculture and an increasingly regulated world. Annual Review of Entomology, 51(1), 45-66.

Isman, M. B. (2008). Botanical insecticides: For richer, for poorer. Pest Management Science, 64(1), 8-11.

Jayaprakasha, G. K., Rao, L. J., \& Sakariah, K. K. (2002). Chemical composition of volatile oil from Cinnamomum zeylanicum buds. Zeitschrift Für Naturforschung C, 57, 990-993.

Jianu, C., Pop, G., Gruia, A. T., \& Horhat, F. G. (2013). Chemical composition and antimicrobial activity of essential oils of lavender (Lavandula angustifolia) and lavandin (Lavandula x intermedia) grown in Western Romania. International Journal of Agriculture and Biology, 15, 772-776.

Jirovetz, L., Buchbauer, G., Denkova, Z., Slavchev, A., Stoyanova, A., \& Schmidt E. (2006). Chemical composition, antimicrobial activities and odor descriptions of various Salvia sp. and Thuja sp. essential oils. Nutrition, 30(4), 152-159.

Kaufmann, C., \& Briegel, H. (2004). Flight performance of the malaria vectors Anopheles gambiae and Anopheles atroparous. Journal of Vector Ecology, 29(1), 140-153.

Klocke, J. A. (1989). Plant compounds as sources and models of insect-control agents. In: Wagner, H., Hikino, H., Farnsworth, N. (Eds.). Economic and medicinal plant research. Academic Press. Vol. 3. Pp. 104-144.

Kordali, S., Aslan, I., Çalmaşur, O., \& Cakir, A. (2006). Toxicity of essential oils isolated from three Artemisia species and some of their major components to granary weevil, Sitophilus granarius (L.) (Coleoptera: Curculionidae). Industrial Crops and Products, 23(2), 162-170.

Koul, O., \& Walia, S. (2009). Comparing impacts of plant extracts and pure allelochemicals and implications for pest control. CAB Reviews: Perspectives in Agriculture, Veterinary Science, Nutrition and Natural Resources, 4, 49.

Koul, O., Walia, S., \& Dhaliwal, G. S. (2008). Essential oils as green pesticides: Potential and constraints. Biopesticides International, 4, 63-84. 
Kuusik, A., Harak, M., Hiiesaar, K., Metspalu, L., \& Tartes, U. (1995). Studies on insect growth regulating (IGR) and toxic effects of Ledum palustre extracts on Tenebrio molitor pupae (Coleoptera, Tenebrionidae) using calorimetric recordings. Thermochimica Acta, 251, 247-253.

Lamiri, A., Lhaloui, S., Benjilali, B., \& Berrada, M. (2001). Fumigant toxic activity of essential oils on Sitophilus granarius (Linné). Physical and Chemical News, 1, 101-105.

Lee, B.-H., Choi, W.-S., Lee, S.-E., \& Park, B.-S. (2001). Fumigant toxicity of essential oils and their constituent compounds towards the rice weevil, Sitophilus oryzae (L.). Crop Protection, 20(4), 317-320.

Lee, S., Najiah, M., Wendy, W., \& Nadirah, M. (2009). Chemical composition and antimicrobial activity of the essential oil of Syzygium aromaticum flower bud (Clove) against fish systemic bacteria isolated from aquaculture sites. Frontiers of Agriculture in China, 3(3), 332-336.

Lee, S.-J., Umano, K., Shibamoto, T., \& Lee, K.-G. (2005). Identification of volatile components in basil (Ocimum basilicum L.) and thyme leaves (Thymus vulgaris L.) and their antioxidant properties. Food Chemistry, 91(1), 131-137.

Lopes-Lutz, D., Alviano, D. S., Alviano, C. S., \& Kolodziejczyk, P. P. (2008). Screening of chemical composition, antimicrobial and antioxidant activities of Artemisia essential oils. Phytochemistry, 69(8), 1732-1738.

Loudon, C. (1988). Development of Tenebrio molitor in low oxygen levels. Journal of Insect Physiology, 34(2), 97-103.

Mahmoudvand, M., Abbasipour, H., Basij, M., Hossein Hosseinpour, M., Rastegar, F., \& Bagher Nasiri, M. (2011). Fumigant toxicity of some essential oils on adults of some stored-product pests. Chilean Journal of Agricultural Research, 71(1), 83-89.

Mann, S. R., \& Kaufman, E. P. (2012). Natural product pesticides: Their development, delivery and use against insect vectors. Mini-Reviews in Organic Chemistry, 9(2), 185-202.

Martínez, L. C., Plata-Rueda, A., Colares, H. C., Campos, J. M., Dos Santos, M. H., Fernandes, F. L., \& Zanuncio, J. C. (2017). Toxic effects of two essential oils and their constituents on the mealworm beetle, Tenebrio molitor. Bulletin of Entomological Research, 108(6), 716-725.

Martynov, V. O., \& Brygadyrenko, V. V. (2017). The influence of synthetic food additives and surfactants on the body weight of larvae of Tenebrio molitor (Coleoptera, Tenebrionidae). Biosystems Diversity, 25(3), 236-242.

Martynov, V. O., \& Brygadyrenko, V. V. (2018). The impact of some inorganic substances on change in body mass of Tenebrio molitor (Coleoptera, Tenebrionidae) larvae in a laboratory experiment. Folia Oecologica, 45(1), 24-32.

Martynov, V. O., Titov, O. G., Kolombar, T. M., \& Brygadyrenko, V. V. (2019). Influence of essential oils of plants on the migration activity of Tribolium confusum (Coleoptera, Tenebrionidae). Biosystems Diversity, 27(2), 120-128.

Mechergui, K., Coelho, J. A., Serra, M. C., Lamine, S. B., Boukhchina, S., Khouja, M. L. (2010). Essential oils of Origanum vulgare L. subsp. glandulosum (Desf.) Ietswaart from Tunisia: Chemical composition and antioxidant activity. Journal of the Science of Food and Agriculture, 90(10), 1745-1749.

Mohamed, M. I. E., \& Abdelgaleil, S. A. M. (2008). Chemical composition and insecticidal potential of essential oils from Egyptian plants against Sitophilus oryzae (L.) (Coleoptera: Curculionidae) and Tribolium castaneum (Herbst) (Coleoptera: Tenebrionidae). Applied Entomology and Zoology, 43(4), 599-607.

Nassar, M. I., Gaara, A. H., El-Ghorab, A. H., Farrag, A.-R. H., Shen, H., Huq, E., \& Mabry, T. J. (2007). Chemical constituents of clove (Syzygium aromaticum, Fam. Myrtaceae) and their antioxidant activity. Revista Latinoamericana de Quimica, 35(3), 47-57.

Neethirajan, S., Karunakaran, C., Jayas, D. S., \& White, N. D. G. (2007). Detection techniques for stored-product insects in grain. Food Control, 18(2), 157-162.

Nikolić, M., Glamočlija, J., Ferreira, I. C. F. R., Calhelha, R. C., Fernandes, Â., Marković, T., \& Soković, M. (2014). Chemical composition, antimicrobial, antioxidant and antitumor activity of Thymus serpyllum L., Thymus algeriensis Boiss. and Reut and Thymus vulgaris L. essential oils. Industrial Crops and Products, $52,183-190$

Özcan, M. M., \& Chalchat, J.-C. (2008). Chemical composition and antifungal activity of rosemary (Rosmarinus officinalis L.) oil from Turkey. International Journal of Food Sciences and Nutrition, 59, 691-698.

Pereira, R. P., Boligon, A. A., Appel, A. S., Fachinetto, R., Ceron, C. S., Tanus-Santos, J. E., \& Rocha, J. B. T. (2014). Chemical composition, antioxidant and anticholinesterase activity of Melissa officinalis. Industrial Crops and Products, 53, 3445.

Pérez, S. G., Ramos-López, M. A., Zavala-Sánchez, M. A., Cárdenas-Ortega, N. C. (2010). Activity of essential oils as a biorational alternative to control coleopteran insects in stored grains. Journal of Medicinal Plants Research, 4(25), $2827-2835$.

Perry, N. B., Anderson, R. E., Brennan, N. J., Douglas, M. H., Heaney, A. J., McGimpsey, J. A., \& Smallfield, B. M. (1999). Essential oils from Dalmatian sage (Salvia officinalis L.): Variations among individuals, plant parts, seasons, and sites. Journal of Agricultural and Food Chemistry, 47(5), 2048-2054.

Pimentel, M. A. G., Faroni, L. R. D., Guedes, R. N. C., Sousa, A. H., \& Tótola, M. R. (2009). Phosphine resistance in Brazilian populations of Sitophilus zeamais
Motschulsky (Coleoptera: Curculionidae). Journal of Stored Products Research, 45(1), 71-74.

Pino, J. A., Rosado, A., \& Fuentes, V. (1998). Chemical composition of the leaf oil of Achillea millefolium L. grown in Cuba. Journal of Essential Oil Research, 10(4), 427-428.

Pirzad, A., Alyari, H., Shakiba, M. R., Zehtab-Salmasi, S., \& Mohammadi, A. (2006) Essential oil content and composition of German chamomile (Matricaria chamomilla L.) at different irrigation regimes. Journal of Agronomy, 5(3), 451-455.

Plata-Rueda, A., Martínez, L. C., Santos, M. H. D., Fernandes, F. L., Wilcken, C. F. Soares, M. A., \& Zanuncio, J. C. (2017). Insecticidal activity of garlic essential oil and their constituents against the mealworm beetle, Tenebrio molitor Linnaeus (Coleoptera: Tenebrionidae). Scientific Reports, 7(1), 46406.

Punzo, F., \& Mutchmor, J. A. (1980). Effects of temperature, relative humidity and period of exposure on the survival capacity of Tenebrio molitor (Coleoptera: Tenebrionidae). Journal of the Kansas of Entomological Society, 53, 260-270.

Pushpanathan, T., Jebanesan, A., \& Govindarajan, M. (2008). The essential oil of Zingiber officinalis Linn (Zingiberaceae) as a mosquito larvicidal and repellent agent against the filarial vector Culex quinquefasciatus Say (Diptera: $\mathrm{Cu}$ licidae). Parasitology Research, 102(6), 1289-1291.

Raal, A., Orav, A., \& Gretchushnikova, T. (2014). Composition of the essential oil of the Rhododendron tomentosum Harmaja from Estonia. Natural Product Research, 28(14), 1091-1098.

Radulescu, V., Saviuc, C., Chifiriuc, C., Oprea, E., Ilies, D. C., Marutescu, L., \& Lazar, V. (2011). Chemical composition and antimicrobial activity of essential oil from shoots spruce (Picea abies L.). Revista de Chimie, 62(1), 69-74.

Rajkumar, V., Gunasekaran, C., Christy, I. K., Dharmaraj, J., Chinnaraj, P., \& Paul, C. A. (2019). Toxicity, antifeedant and biochemical efficacy of Mentha piperita L. essential oil and their major constituents against stored grain pest. Pesticide Biochemistry and Physiology, 156, 138-144.

Rezaeinodehi, A., \& Khangholi, S. (2008). Chemical composition of the essential oil of Artemisia absinthium growing wild in Iran. Pakistan Journal of Biological Sciences, 11(6), 946-949.

Rohloff, J., Skagen, E. B., Steen, A. H., \& Iversen, T.-H. (2000). Production of yarrow (Achillea millefolium L.) in Norway: Essential oil content and quality. Journal of Agricultural and Food Chemistry, 48(12), 6205-6209.

Rotimi, O. A., Chris, O. A., Olusola, O. O., Joshua, R., \& Josiah, A. O. (2011) Bioefficacy of extracts of some indigenous Nigerian plants on the developmental stages of mosquito (Anopheles gambiae). Jordan Journal of Biological Sciences, 4(4), 237-242.

Rozman, V., Kalinovic, I., \& Liska, A. (2006). Insecticidal activity of some aromatic plants from Croatia against granary weevil (Sitophilus granarius L.) on stored wheat. Cereal Research Communications, 34(1), 705-708.

Ryan, M. F., \& Byrne, O. (1988). Plant-insect coevolution and inhibition of acetylcholinesterase. Journal of Chemical Ecology, 14(10), 1965-1975.

Saharkhiz, M. J., Motamedi, M., Zomorodian, K., Pakshir, K., Miri, R., \& Hemyari, K. (2012). Chemical composition, antifungal and antibiofilm activities of the essential oil of Mentha piperita L. ISRN Pharmaceutics. Pp. 1-6.

Sandoval-Montemayor, N. E., García, A., Elizondo-Treviño, E., Garza-González, E., Alvarez, L., \& del Rayo Camacho-Corona, M. (2012). Chemical composition of hexane extract of Citrus aurantifolia and anti-Mycobacterium tuberculosis activity of some of its constituents. Molecules, 17(9), 11173-11184.

Schearer, W. R. (1984). Components of oil of tansy (Tanacetum vulgare) that repel Colorado potato beetles (Leptinotarsa decemlineata). Journal of Natural Products, 47(6), 964-969.

Schroeckenstein, D., Meier-Davis, S., \& Bush, R. (1990). Occupational sensitivity to Tenebrio molitor Linnaeus (yellow mealworm). Journal of Allergy and Clinica Immunology, 86(2), 182-188.

Scott, I. M., Jensen, H., Scott, J. G., Isman, M. B., Amason, J. T., \& Philogène, B. J. R. (2003). Botanical insecticides for controlling agricultural pests: Piperamides and the Colorado potato beetle Leptinotarsa decemlineata Say (Coleoptera: Chrysomelidae). Archives of Insect Biochemistry and Physiology, 54(4), 212-225.

Shabby, A. S., El-Gengaihi, S., \& Khattab, M. (1995). Oil of Melissa officinalis L., as affected by storage and herb drying. Journal of Essential Oil Research, 7(6), 667-669.

Singh, G., Kapoor, I. P. S., Singh, P., de Heluani, C. S., de Lampasona, M. P., \& Catalan, C. A. N. (2008). Chemistry, antioxidant and antimicrobial investigations on essential oil and oleoresins of Zingiber officinale. Food and Chemical Toxicology, 46(10), 3295-3302.

Singh, P. Shukla, R, Prakash, B, Kumar, A, Singh, S, Mishra, P. K, \& Dubey, N. K. (2010). Chemical profile, antifungal, antiaflatoxigenic and antioxidant activity of Citrus maxima Burm. and Citrus sinensis (L.) Osbeck essential oils and their cyclic monoterpene, DL-limonene. Food and Chemical Toxicology, 48(6), 1734-1740.

Subasinghe, U., Gamage, M., \& Hettiarachchi, D. S. (2013). Essential oil conten and composition of Indian sandalwood (Santalum album) in Sri Lanka. Journal of Forestry Research, 24(1), 127-130.

Teixeira, B., Marques, A., Ramos, C., Serrano, C., Matos, O., Neng, N. R., \& Nunes, M. L. (2013). Chemical composition and bioactivity of different oregano (Ori- 
ganum vulgare) extracts and essential oil. Joumal of the Science of Food and Agriculture, 93(11), 2707-2714.

Tripathi, A. K., Upadhyay, S., Bhuiyan, M., \& Bhattacharya, P. R. (2009). A review on prospects of essential oils as biopesticide in insect-pest management. Journal of Pharmacognosy and Phytotherapy, 1, 52-63.

Uysal, B., Sozmen, F., Aktas, O., Oksal, B. S., \& Kose, E. O. (2011). Essential oil composition and antibacterial activity of the grapefruit (Citrus paradisi L.) peel essential oils obtained by solvent-free microwave extraction: Comparison with hydrodistillation. International Journal of Food Science and Technology, 46(7), 1455-1461.

Wang, X., Hao, Q., Chen, Y., Jiang, S., Yang, Q., \& Li, Q. (2015). The effect of chemical composition and bioactivity of several essential oils on Tenebrio molitor (Coleoptera: Tenebrionidae). Journal of Insect Science, 15(1), 116.

Wang, Y., Li, P., \& Chi, D. (2016). Electrophysiological and behavioral responses of Tenebrio molitor L. to fourteen kinds of plant volatiles. Journal of AsiaPacific Entomology, 19(2), 261-267.
Weinzierl, R. (1998). Botanical insecticides, soaps, and oils. In: Rechcigl, J. E., \& Rechcigl, N. A. (Eds.). Biological and biotechnological control of insect pests. CRC Press LLC, Boca Raton, New York. Pp. 110-130.

Werdin-González J. O., Murray, A. P \& Ferrero, A A (2008). Bioactividad de aceites esenciales de Schinus molle var. areira (Anacardiaceae) en ninfas II de Nezara viridula (Hemiptera: Pentatomidae). Boletin de Sanidad Vegetal Plagas, 34, 367-375.

Yazdgerdian, A. R., Akhtar, Y., \& Isman, M. B. (2015). Insecticidal effects of essential oils against woolly beech aphid, Phyllaphis fagi (Hemiptera: Aphididae) and rice weevil, Sitophilus oryzae (Coleoptera: Curculionidae). Journal of Entomology and Zoology Studies, 3(3), 265-271.

Yildrim, E., Kesdek, M., Asian, I., Calmasur, O., \& Sahin, F. (2005). The effect of essential oils from eight plant species on two pests of stored product insects. Fresenius Environmental Bulletin, 15(1), 23-27. 